\title{
Estimation of aquifer lower layer hydraulic conductivity values through base flow hydrograph rising limb analysis
}

\author{
Valentijn R. N. Pauwels ${ }^{1}$ and Peter A. Troch ${ }^{2}$ \\ Received 29 May 2009; revised 24 September 2009; accepted 1 October 2009; published 3 March 2010.
}

[1] The estimation of catchment-averaged aquifer hydraulic conductivity values is usually performed through a base flow recession analysis. Relationships between the first time derivatives of the base flow and the base flow values themselves, derived for small and large values of time, are used for this purpose. However, in the derivation of the short-time equations, an initially fully saturated aquifer without recharge with sudden drawdown is assumed, which occurs very rarely in reality. It is demonstrated that this approach leads to a nonnegligible error in the parameter estimates. A new relationship is derived, valid for the rising limb of a base flow hydrograph, succeeding a long rainless period. Application of this equation leads to accurate estimates of the aquifer lower layer saturated hydraulic conductivity. Further, it has been shown analytically that, if base flow is modeled using the linearized Boussinesq equation, the base flow depends on the effective aquifer depth and the ratio of the saturated hydraulic conductivity to the drainable porosity, not on these three parameters separately. The results of the new short-time expression are consistent with this finding, as opposed to the use of a traditional base flow recession analysis. When base flow is modeled using the nonlinear Boussinesq equation, the new expression can be used, without a second equation for large values of time, to estimate the aquifer lower layer hydraulic conductivity. Overall, the results in this paper suggest that the new methodology outperforms a traditional recession analysis for the estimation of catchment-averaged aquifer hydraulic conductivities.

Citation: Pauwels, V. R. N., and P. A. Troch (2010), Estimation of aquifer lower layer hydraulic conductivity values through base flow hydrograph rising limb analysis, Water Resour. Res., 46, W03501, doi:10.1029/2009WR008255.

\section{Introduction}

[2] The representation of groundwater flow processes in hydrologic models is usually referred to as the horizontal or lateral redistribution of soil water [Wood, 1991]. Even though at small spatial scales these processes cannot automatically be neglected, in many models, applied at large (continental) scales, these processes are not represented. An example of a widely used model that does not account for lateral redistribution is the Common Land Model (CLM) [Dai et al., 2003]. On the other hand, the Richards equation [Richards, 1931] can be solved numerically in a full three-dimensional manner, as is demonstrated by, for example, Paniconi and Wood [1993] and Camporese et al. [2009]. This methodology has the advantage that the horizontal redistribution of soil water is modeled in a physically based way. The drawbacks are that a large number of spatially distributed parameter values are required, that the computational demands can render this approach infeasible for larger modeling domains, and that effective parameter values cannot be determined in the

\footnotetext{
${ }^{1}$ Laboratory of Hydrology and Water Management, Ghent University, Ghent, Belgium.

${ }^{2}$ Department of Hydrology and Water Resources, University of Arizona, Tucson, Arizona, USA.
}

Copyright 2010 by the American Geophysical Union. 0043-1397/10/2009WR008255\$09.00 presence of heterogeneity. If one assumes the aquifers to be homogeneous and isotropic (a reasonable assumption if one wants a relatively simple model, taking into account all other uncertainties), the need for large parameters sets is strongly reduced, but the computational demands can in many cases still be excessive. A middle of the road approach is to model the saturated groundwater flow processes in a lumped manner, meaning that the lateral redistribution of soil water is modeled using simple methods that are obtained using a variety of assumptions, strongly reducing the required computer time. Examples of models that apply this approach are the Variable Infiltration Capacity (VIC) model [Liang et al., 1994] and the TOPMODEL-based Land Atmosphere Transfer Scheme [Famiglietti and Wood, 1994].

[3] For the latter approach, aquifer hydraulic parameters, such as the saturated hydraulic conductivity, are required at the catchment scale. Although in theory these parameters can be measured in laboratories using soil samples, the difference between the scale of the observations and the scale at which the processes occur renders these measurements inadequate for application in hydrologic models [Bear, 1972]. For this reason, methods have been developed, in which these parameters are estimated through a hydrograph recession analysis. More specifically, using theoretically derived equations, the rate of the recessions can be related to the aquifer hydraulic parameters. In order to eliminate the time variable in these relationships, the discharge recessions are analyzed as a 
function of the discharge [Brutsaert and Nieber, 1977; Troch et al., 1993; Rupp and Selker, 2005; Huyck et al., 2005]. Usually, approximations for short and large times are derived. Based on these expressions, the aquifer parameters can be related to observed recession dynamics. Rupp and Selker [2006a] provide an overview of such relationships, including the assumptions used in their derivation. These assumptions apply to the slope of the aquifer, the linearization of the governing equation, the incorporation of variable geometry, the uniformity of the aquifer parameters, and the omission of certain terms in the governing equation.

[4] In the application of the short-time equations, a number of inconsistencies are usually neglected. For example, although conditions of probable full saturation have been documented for an isolated hydrograph caused by extreme rainfall for a small catchment [Lyon et al., 2008], full saturation combined with zero recharge at the onset of a recession and a sudden drawdown at the bottom of the aquifer is a condition that arguably occurs very rarely in reality. Further, it is known that hydraulic conductivity values are not uniform over the soil profile [Beven and Kirkby, 1979; Rupp and Selker, 2005, 2006a]. It can be expected that the inconsistency between the conditions under which the equations have been derived and the conditions under which they are applied will lead to errors in the parameter estimates.

[5] The objective of this paper is to develop a methodology to estimate catchment-scale hydraulic conductivity values of the lower layers of the underlying aquifer, consistent with the conditions under which the required equations are derived. Such estimates are crucial to quantify catchment behavior during drought periods when aquifer storage is near minimum. To develop such a method, the theory related to the governing equation (the extended Boussinesq equation) is first summarized. A general equation for the short-time behavior of the base flow, valid for realistic initial conditions and recharge rates, is derived using the linearized extended Boussinesq equation. This expression is further simplified for the case of the rising limb of a base flow hydrograph succeeding a long rainless period. It should be noted that, recently, advances have been made in the estimation of catchment averaged net rainfall rates using fluctuations in streamflow time series [Kirchner, 2009]. However, the methodology developed in this paper requires time series of base flow instead of streamflow. Instead of estimating catchment averaged net rainfall rates, in this paper it is demonstrated that the rising limbs of base flow hydrographs can be related to the hydraulic properties on an aquifer. Synthetic experiments are used to demonstrate the accuracy of this new equation, and to assess the impact of the unrealistic assumptions in the traditionally used expressions for the short-time behavior of base flow. The results of a traditional base flow recession analysis are compared to the results of the newly developed methodology. Further, a dimensional analysis is performed, for the purpose of assessing whether the parameter sensitivity of the newly derived short-time expression is consistent with the parameter sensitivity of the linearized extended Boussinesq equation. A suggestion is then made in order to apply the new equation for the estimation of aquifer hydraulic conductivities, in case the nonlinear extended Boussinesq equation is used. The paper finally concludes with a discussion on the strengths and weaknesses of the newly developed methodology, based on the use of the rising limbs of hydrographs, versus a traditional recession analysis.

\section{Methodology}

[6] In order to develop a methodology to determine catchment-scale aquifer hydraulic conductivity values, consistent with the conditions under which the required equations are derived, the following approach is used. First, an expression describing the behavior of the base flow for short times during a recharge event is derived. This expression is then validated using a synthetic time series of recharge, applied to an aquifer with predefined parameters. This analysis will allow an interpretation of the applicability of the new short-time solution.

[7] As a next step, the aquifer hydraulic parameters obtained using a traditional base flow recession analysis are compared to the parameters obtained using the new method. For this purpose, time series of recharge rates and base flow values will be used that were generated for the Zwalm catchment in Belgium. Pauwels et al. [2002] describe how these time series were generated. To summarize, the net precipitation (the precipitation leading to discharge) was obtained by multiplying the precipitation by a monthly averaged runoff coefficient. A fraction of this net precipitation was assumed to infiltrate, not allowing the infiltration rate to exceed a specified maximum value. The infiltration rate was used as recharge to the groundwater table, of which the outflow was calculated using equation (13) in section 3.1, the general expression for the flow rate out of the aquifer. In order to apply this equation, the catchment was conceptualized as two aquifers, draining into the stream network of the catchment. The topographic parameters for these aquifers (width, length, and slope) were obtained through a Digital Elevation Model analysis. The aquifer width was obtained by dividing the catchment area by twice the total stream length (since two aquifers with equal properties are draining into the stream network). The aquifer slope was assumed to be equal to the average slope of the topography in the catchment. The hydraulic parameters for the model were obtained through a traditional base flow recession analysis [Brutsaert and Nieber, 1977]. The surface runoff was routed to the outlet of the basin using the unit hydrograph approach of Troch et al. [1994].

[8] In order to generalize the results, the base flow is also modeled through a numerical solution to the nonlinear equation (equation (4) in section 3.1). A very simple model was thus used for this purpose. For the objectives of the study, the model was deemed to be sufficiently detailed, since this paper focuses on parameter retrieval, not hydrologic model development. Inclusion of, for example, unsaturated zone processes, may be more realistic, but will not alter the conclusions drawn in the paper.

[9] Using this simple model, the base flow is generated in a manner consistent with the equations developed in this study. If the modeled discharge is comparable to the observations, it can be assumed that the infiltration rates and consequently the groundwater recharge rates are sufficiently realistic to be used in a synthetic experiment. The resulting base flow values will then be used to assess the applicability 


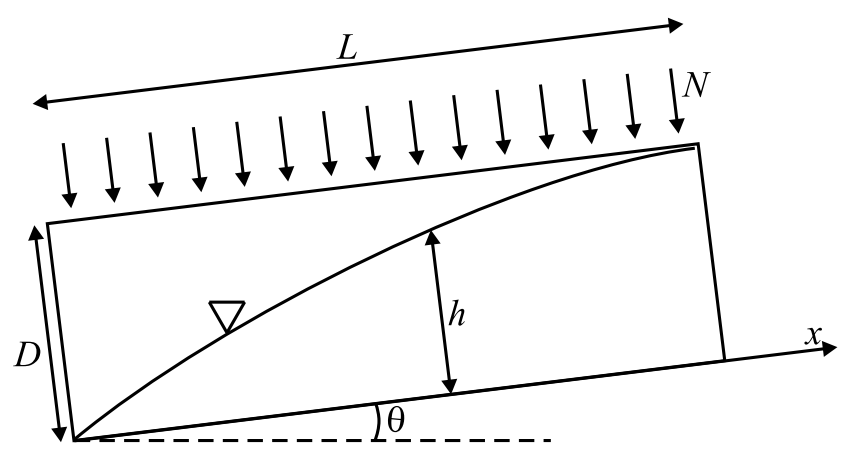

Figure 1. Schematic representation of the parameters used in the Boussinesq equation. The length scale is smaller than the height scale; typically, the length of the aquifer is an order of magnitude larger than the height.

of the approximative solutions to the Boussinesq equation developed in section 3 .

[10] It should be noted that the applicability of the new methodology is thus assessed through a synthetic experiment. The reason for this approach is that, even if a measured time series of base flow and recharge rates would be available with very high accuracy, it is still not possible to directly measure the catchment-averaged aquifer hydraulic parameters. It would thus be impossible to validate the retrieved value of, for example, the hydraulic conductivity. On the other hand, if a time series of base flow is generated using a known time series of recharge rates, using the same governing equation upon which the methodology is based, the same hydraulic parameters as were used in the modeling of the base flow should be retrieved. A synthetic experiment is thus a better way to validate the methodology developed in this paper. It is thus irrelevant that the hydraulic parameters used in the hydrologic model are obtained through a traditional base flow recession analysis. The important aspect of the paper is that the original parameter values are retrieved by the methodology developed in this paper.

\section{Theoretical Derivation}

\subsection{Governing Equation}

[11] In a traditional base flow recession analysis, the expressions relating the first time derivative of the base flow to the base flow are obtained through analytical solutions to the Boussinesq equation. This equation is derived through a combination of the Darcy equation and the continuity equation, using hydraulic ground water theory and strictly onedimensional subsurface flow. In this paper, the nonlinear and linearized versions of the extended Boussinesq equation are used. The term extended means that the aquifer width does not have to be uniform. The details regarding this derivation are given by Troch et al. [2002, 2003] and Huyck et al. [2005]. Only a short summary will be provided here.

[12] Figure 1 shows a schematic overview of an inclined hillslope, indicating the symbols used in the derivation of the extended Boussinesq equation. The assumption is made that the width of the aquifer $W(\mathrm{~m})$ varies exponentially as a function of the distance to the river [Fan and Bras, 1998]:

$$
W(x)=\alpha e^{-\beta x}
$$

$\beta$ is the aquifer shape parameter $\left(\mathrm{m}^{-1}\right), \alpha$ is the width of the aquifer at the outlet (m), and $x$ is the distance in the aquifer to the outlet $(\mathrm{m})$. Figure 2 shows the aquifer shape for different values of $\beta$. Using a homogeneous drainable porosity $f$ (dimensionless), the continuity equation can be written as [Troch et al., 2003]

$$
f W(x) \frac{\partial h}{\partial t}+\frac{\partial(W(x) q(x))}{\partial x}=W(x) N
$$

$h$ is the elevation of the groundwater table measured perpendicular to the underlying impermeable layer $(\mathrm{m}), t$ is the time (s), $q$ is the outflow per unit width $\left(\mathrm{m}^{2} \mathrm{~s}^{-1}\right), f$ is the drainable porosity (dimensionless), and $N$ is the recharge uniformly distributed across the aquifer $\left(\mathrm{m} \mathrm{s}^{-1}\right)$. With the Dupuit-Forchheimer assumption (hydraulic head is independent of depth), and the additional assumption that the streamlines are parallel to the bed, the Darcy equation is given by

$$
q=-k h\left(\frac{\partial h}{\partial x} \cos \theta+\sin \theta\right)
$$

provided that the aquifer is homogeneous and isotropic. $k$ is the saturated hydraulic conductivity $\left(\mathrm{m} \mathrm{s}^{-1}\right)$, and $\theta$ the aquifer slope (radians). Combination of equations (1), (2), and (3) leads to

$$
\begin{aligned}
\frac{\partial h}{\partial t}= & \frac{k}{f}\left(\frac{\partial h}{\partial x}\right)^{2} \cos \theta+\frac{k}{f} h \frac{\partial^{2} h}{\partial x^{2}} \cos \theta+\frac{k}{f} \frac{\partial h}{\partial x} \sin \theta \\
& -\frac{k}{f} h \beta\left(\frac{\partial h}{\partial x} \cos \theta+\sin \theta\right)+\frac{N}{f}
\end{aligned}
$$

This is the nonlinear extended Boussinesq equation, which can be solved numerically. In order to allow an analytical
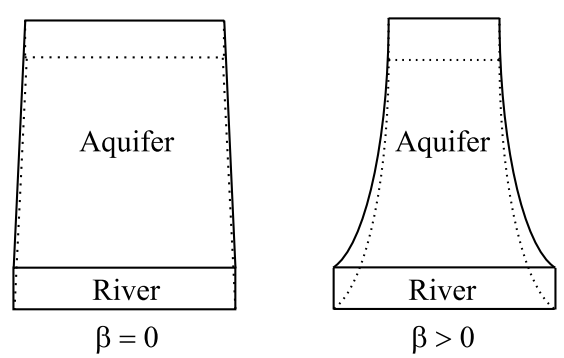

Figure 2. Shape of the aquifer for different values of $\beta$. 


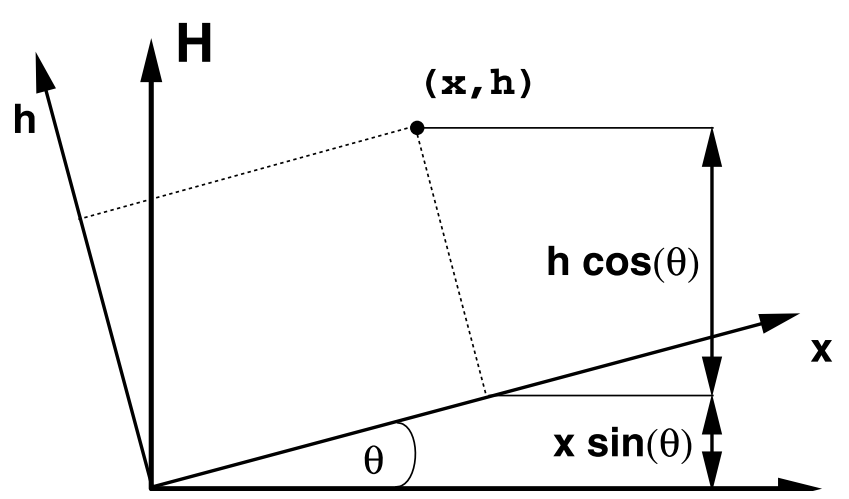

Figure 3. Transformation of the coordinates $(x, h)$ in a sloping aquifer to a horizontal reference surface.

solution, the linearization technique of Brutsaert [1994] can be applied to equation (3):

$$
q=-k p D \cos \theta \frac{\partial h}{\partial x}-k h \sin \theta
$$

$D$ is the aquifer depth $(\mathrm{m})$, and $p$ the linearization constant $(-)$. The parameters $K\left(\mathrm{~m}^{2} \mathrm{~s}^{-1}\right)$ and $U\left(\mathrm{~m} \mathrm{~s}^{-1}\right)$ are introduced:

$$
\left\{\begin{array}{l}
K=\frac{k p D \cos \theta}{f} \\
U=\frac{k \sin \theta}{f}
\end{array}\right.
$$

Using these, the linearized Darcy equation can be written as

$$
q=-K f \frac{\partial h}{\partial x}-U f h
$$

Other linearization techniques are described by Chapman [1995] and Troch et al. [2004]. However, in this paper, the technique of Brutsaert [1994] was used, since for this technique the most advanced analytical solutions exist. Combining the linearized Darcy equation (equation (7)) with the continuity equation (equations (2) and (1)) results in the linearized extended Boussinesq equation:

$$
\frac{\partial h}{\partial t}=K \frac{\partial^{2} h}{\partial x^{2}}+(U-\beta K) \frac{\partial h}{\partial x}-\beta U h+\frac{N}{f}
$$

This is the equation which is solved analytically in this paper for a number of specific limiting cases.

\subsection{Physical Interpretation of the Linearization}

[13] A thorough understanding of the linearization technique applied to equation (3) is important for a correct interpretation of the analytical solutions to equation (8). For this purpose, the assumptions used in the derivation of equation (3) need to be discussed. Figure 3 shows a schematic of the variables used for this purpose. The major assumption is that the streamlines are parallel to the impermeable layer. When the hydraulic conductivity is uniform with depth, this implies that the transmissivity of the aquifer is equal to the saturated hydraulic conductivity $k$ multiplied by the water level $h$, measured perpendicular to the bed- rock. In order to obtain the flow rate, this transmissivity needs to be multiplied by the gradient in the water level, measured perpendicular to the horizontal reference surface. The flow rate thus becomes

$$
q=-k h \frac{\partial H}{\partial x}
$$

$H$ is the height of the water table, measured perpendicular to the horizontal reference surface $(\mathrm{m})$. Figure 3 shows that the water level $H$ can easily be transformed to the coordinate system with slope $\theta$ (the aquifer slope) as follows:

$$
H=h \cos \theta+x \sin \theta
$$

If the aquifer slope is zero (which is only a limit case used for completeness), the flow rate can be written as

$$
q=-k h \frac{\partial h}{\partial x}
$$

If the linearization technique of Brutsaert [1994] is used, the flow rate becomes

$$
q=-k p D \frac{\partial h}{\partial x}
$$

It is thus easy to see that in this case $k p D$ is the transmissivity of the aquifer $\left(\mathrm{m}^{2} \mathrm{~s}^{-1}\right), p D$ is defined as the effective aquifer depth $(\mathrm{m})$, which is the depth of the aquifer through which groundwater flows. Ideally, if the aquifer is relatively saturated, $p$ should be chosen close to 1 , and for a relatively dry aquifer, $p$ should approximate zero. Koussis [1992] and Rupp and Selker [2006a] provide a theoretical basis to estimate $p$. However, analytical solutions to equation (8) for variable values of $p$ are extremely difficult to obtain. For this reason, a compromise is usually made, in which $p$ is chosen between $1 / 2$ and $1 / 3$ [Brutsaert, 1994]. In this paper, if analytical solutions to equation (8) are analyzed, a value of $1 / 2$ is used. For aquifers with nonzero slope, a similar interpretation can be made. For example, for a slope of 0.147 radians (which is the slope of the study area), the expression for $q$ becomes $-0.75 \mathrm{kpD \partial h} / \partial x-$ $0.25 h$. At $x$ equal to zero, $h$ is equal to zero, so again $k p D$ can be assumed to be an effective transmissivity. Under relatively steep slopes, the Boussinesq equation thus still represents the processes of advection and diffusion. A different approach, the kinematic wave, in which the first term is neglected, only includes the process of advection. For the purposes of this study, it is thus better to use the Boussinesq equation as theoretical basis, as opposed to other approximations.

\subsection{Analytical Solution}

[14] Huyck et al. [2005] provide a general solution to equation (8), under the assumptions of a zero water level at the aquifer outlet, and a zero flux at the hillcrest. A number of studies have focused on the assumption of a zero water level at the aquifer outlet [Chapman, 2005; Chapman and Ong, 2006]. However, Pauwels et al. [2002] demonstrated that the value of a nonzero water level does not appear in the analytical expression for the base flow, which justifies this assumption for the purpose of this study. The above 
boundary conditions have been used in a number of studies, including Brutsaert [1994], Verhoest and Troch [2000], Pauwels et al. [2002], Troch et al. [2003], Paniconi et al. [2003], Troch et al. [2004], and Hilberts et al. [2004].

[15] The aquifer is subject to a series of recharge rates $N_{1}$, $N_{2}, \ldots, N_{m}\left(\mathrm{~m} \mathrm{~s}^{-1}\right)$, each with duration $T_{1}, T_{2}, \ldots, T_{m}(\mathrm{~s})$. The initial condition is a steady state profile obtained using the recharge rate $N_{0}$. Under these conditions, the base flow rate out of the basin $\left(Q_{b}, \mathrm{~m}^{3} \mathrm{~s}^{-1}\right)$ can be written as [Huyck et al., 2005]

$$
\begin{aligned}
Q_{b}(t)= & A N_{m}+\left(N_{m}-N_{m-1}\right)\left[\sum_{n=1}^{\infty} \frac{4 W_{0} K Z_{n}}{U \beta+y_{n}} \frac{z_{n}}{L} e^{y_{n} t-a L}\right] \\
& +\sum_{i=1}^{m-1}\left(N_{i}-N_{i-1}\right)\left[\sum_{n=1}^{\infty} \frac{4 W_{0} K Z_{n}}{U \beta+y_{n}} \frac{z_{n}}{L} e^{y_{n}\left(t+\mathcal{T}_{i}\right)-a L}\right]
\end{aligned}
$$

$A$ is the area of the catchment $\left(\mathrm{m}^{2}\right)$, and $W_{0}$ is the total stream length in the catchment $(\mathrm{m}) . a\left(\mathrm{~m}^{-1}\right), z_{n}$ (dimensionless), $y_{n}\left(\mathrm{~s}^{-1}\right), Z_{n}$ (dimensionless), and $\mathcal{T}_{i}(\mathrm{~s})$ are calculated as

$$
\left\{\begin{aligned}
a & =-\frac{U-K \beta}{2 K} \\
\tan z_{n} & =-\frac{z_{n}}{L(\beta-a)} \\
y_{n} & =-K\left(a^{2}+\frac{z_{n}^{2}}{L^{2}}\right)-U \beta \\
Z_{n} & =z_{n} \frac{e^{a L}+\frac{2 a-\beta}{\beta-a} \cos z_{n}}{L^{2}(\beta-a)^{2}+L(\beta-a)+z_{n}^{2}} \\
\mathcal{T}_{i} & =\sum_{j=1}^{m-1} T_{j}
\end{aligned}\right.
$$

$z_{n}$ are the infinite number of roots of the second expression in equation (14). Appendix A shows the details of the derivation of this equation.

[16] Under the conditions of an initially saturated aquifer, under zero recharge, Huyck et al. [2005] derived the following relationship between the first derivative of the discharge with respect to time and the discharge, for small values of $t$ :

$$
-\frac{d Q_{b}(t)}{d t}=\frac{\pi}{8 W_{0}^{2} k D^{3} f p \cos \theta}\left(Q_{b}(t)\right)^{3}
$$

It should be noted that the relationship between the intercept and the aquifer parameters $W_{0}, K, k$, and $f$, is similar as for the expressions obtained by Polubarinova-Kochina [1962], Lockington [1997], and Rupp and Selker [2005], and is identical to the expression obtained by Brutsaert [1994]. Rupp and Selker [2005] demonstrated that the time window in which equation (15) is valid, reduces as the aquifer slope increases. However, in this paper, a new equation valid for short times will be derived, and it will be demonstrated that this equation can also be used for aquifers with a relatively steep slope. On the other hand, for equation (13) this relationship becomes, for large values of $t$ [Huyck et al., 2005]:

$$
-\frac{d Q_{b}(t)}{d t}=y_{1} Q_{b}(t)
$$

In a logarithmic plot with the discharge values in abscissa ( $x$ axis), and the first derivatives in ordinate ( $y$ axis), equation (15) represents a straight line with slope 3 , and equation (16) a straight line with slope 1 . It should be noted that the slope of 1 is a consequence of the linearization of the governing equation. For the nonlinear equation, in theory, a slope of 1.5 should be obtained [Troch et al., 1993]. Deviations from this theoretic slope can be explained by heterogeneity in the parameter values [Rupp and Selker, 2006a; Harman and Sivapalan, 2009a]. If an estimate of $f$ is available, $k$ and $p D$ can be calculated using the intercepts of these two straight lines.

\subsection{Impact of Parameter Heterogeneity}

[17] In the application of equation (4) or equation (8), two kinds of heterogeneity need to be taken into account, more specifically vertical and horizontal heterogeneity in the aquifer parameter values.

[18] Rupp and Selker [2006a] argue that hydraulic properties often vary with depth. However, both equations (15) and (16) have been derived under the assumption of vertically invariant aquifer hydraulic parameters. In this context, it is important to note that this paper focuses on the estimation of aquifer lower layer hydraulic conductivity values. It is reasonable to assume that for these thin layers the aquifer properties are homogeneous with depth. For the test site used in this study, sedimentation is the main source of vertical heterogeneity, which justifies the assumption of vertical homogeneity used in this paper.

[19] Another aspect to consider is horizontal heterogeneity of the hydraulic parameters in the catchment. Szilagyi et al. [1998] suggested, using lognormally distributed hydraulic conductivity fields, that a recession analysis under these conditions performed equally well as for a homogeneous catchment. However, as is discussed by Harman and Sivapalan [2009a], horizontal heterogeneity can be the explanation of why the exponent in the discharge recessions for a catchment can reach high values (up to two). Further, Harman et al. [2009] demonstrate that, even if individual hillslopes demonstrate linear behavior, the integration of the response of multiple heterogeneous hillslopes can lead to nonlinear behavior in the storage-discharge relationship.

[20] The combined effect of both types of heterogeneity is that, if the linearized Boussinesq equation is analyzed, the slope in the relationship between the time derivative of the base flow and the base flow will deviate from one. However, in order to test whether a slope of one in the recession data is obtained, the twenty years of data from Huyck et al. [2005] were reanalyzed. The approach of Rupp and Selker [2006b] was applied, in order to ensure that artifacts in the data were removed. Only the data points which were preceded by at least four dry days were analyzed, in order to maximize the probability that the solution for large times is valid for these points. A linear regression through the logarithmic plot resulted in a slope of 1.07, which can be considered sufficiently close to 1 for the linearized Boussinesq equation to be valid.

\subsection{Realistic Expression for Short Times}

[21] Arguably, equation (15) has been obtained using unrealistic initial conditions, more specifically, an initially 
fully saturated aquifer with zero recharge, which in reality does not occur frequently. Therefore, a different expression will be derived here, that is obtained in the same manner as equation (13), but which is valid for short times during a recharge event. More specifically, equation (13) has been derived through a solution to equation (8). This equation is solved by Huyck et al. [2005] using Laplace transformation. Appendix A summarizes this derivation. If the aquifer is subjected to a recharge rate $N_{2}$, preceded by a recharge rate $N_{1}$ with duration $T_{1}$, starting from a steady state initial condition obtained with recharge rate $N_{0}$, the Laplace transform of the solution can be written as (equation (A15))

$$
\begin{aligned}
F= & \frac{U \beta N_{2}+N_{1} y}{y f U \beta(U \beta+y)}+N_{1} \frac{(2 a-\beta)}{2 y f U \beta(\beta-a) e^{\beta L}} e^{\beta x} \\
& -N_{1} \frac{2(\beta-a) e^{\beta L}+(2 a-\beta)}{2 y f U \beta(\beta-a) e^{\beta L}} e^{(2 a-\beta) x} \\
& +2 \sum_{n=1}^{\infty} \frac{N_{1}-N_{0}}{f\left(U \beta+y_{n}\right)} Z_{n} \frac{\sin \left(\frac{z_{n} x}{L}\right) e^{y_{n} T_{1}-a(L-x)}}{y-y_{n}} \\
& +\frac{N_{2}-N_{1}}{f(U \beta+y) y} \\
& +\frac{\left[(\beta-a-b) e^{a(L+x)+b(x-L)}-(\beta-a+b) e^{a(L+x)+b(L-x)}-2 e^{a x}(-2 a+\beta) \sinh (b x)\right]}{2 e^{a L}((\beta-a) \sinh (b L)+b \cosh (b L))}
\end{aligned}
$$

The limit for $y$ approaching infinity is, again for $x$ equal to zero:

$$
\begin{aligned}
\lim _{y \rightarrow \infty} \frac{d F}{d x}= & (2 a-\beta) N_{1} \frac{1-e^{\beta L}}{y f U \beta e^{\beta L}}+2 \sum_{n=1}^{\infty} \frac{N_{1}-N_{0}}{f\left(U \beta+y_{n}\right)} Z_{n} \frac{z_{n}}{L} \\
& \cdot \frac{e^{y_{n} T_{1}-a L}}{y-y_{n}}+\frac{N_{2}-N_{1}}{f} \frac{1}{\sqrt{K y^{3}}}
\end{aligned}
$$

Application of equation (19) and multiplication by $-2 W_{0}$ (the minus sign is to render base flow values positive, and $2 W_{0}$ where $y$ is the Laplace variable, and $F$ is the Laplace transform of $h ; b$ is written as

$$
b=\sqrt{a^{2}+\frac{U \beta+y}{K}}
$$

The Laplace transform of the base flow can then be written as [Huyck et al., 2005]

$$
q_{L}(y)=-f K \frac{d F}{d x}-f U F
$$

The limit case for times approaching zero is equivalent to values for $y$ approaching infinity. For equation (17) this limit becomes, for $x$ equal to zero:

$$
\lim _{y \rightarrow \infty} F=-\frac{N_{2}-N_{1}}{f y^{2}}
$$

The first derivative of $F$ with respect to $x$ is accounts for the length of the drainage network with drainage from both banks) leads to the Laplace transform of the base flow for the catchment:

$$
\begin{aligned}
Q_{L}(y)= & 2 W_{0} U \frac{N_{2}-N_{1}}{y^{2}}+2 W_{0} K(2 a-\beta) N_{1} \frac{1-e^{\beta L}}{y U \beta e^{\beta L}} \\
& +4 W_{0} K \sum_{n=1}^{\infty} \frac{N_{1}-N_{0}}{U \beta+y_{n}} Z_{n} \frac{z_{n}}{L} \frac{e^{y_{n} T_{1}-a L}}{y-y_{n}}+2 W_{0}\left(N_{2}-N_{1}\right) \sqrt{\frac{K}{y^{3}}}
\end{aligned}
$$

We neglect the first term in equation (23), since it is divided by $y^{2}$, as opposed to the other terms, which are divided by $y$ to a lower power. We know that $(2 a-\beta) K$ is equal to $-U$ (equation (6)). We thus get

$$
\begin{aligned}
Q_{L}(y)= & -2 W_{0} \frac{N_{1}}{\beta y}\left(e^{-\beta L}-1\right)+4 W_{0} K \sum_{n=1}^{\infty} \frac{N_{1}-N_{0}}{U \beta+y_{n}} Z_{n} \frac{z_{n}}{L} \frac{e^{y_{n} T_{1}-a L}}{y-y_{n}} \\
& +2 W_{0}\left(N_{2}-N_{1}\right) \sqrt{\frac{K}{y^{3}}}
\end{aligned}
$$

$$
\begin{aligned}
\frac{d F}{d x}= & N_{1} \frac{(2 a-\beta) \beta}{2 y f U \beta(\beta-a) e^{\beta L}} e^{\beta x}-N_{1}(2 a-\beta) \frac{2(\beta-a) e^{\beta L}+(2 a-\beta)}{2 y f U \beta(\beta-a) e^{\beta L}} e^{(2 a-\beta) x} \\
& +2 \sum_{n=1}^{\infty} \frac{N_{1}-N_{0}}{f\left(U \beta+y_{n}\right)} Z_{n} \frac{z_{n} \frac{\cos \left(\frac{z_{n} x}{L}\right) e^{y_{n} T_{1}-a(L-x)}}{y-y_{n}}}{} \\
& +2 \sum_{n=1}^{\infty} \frac{N_{1}-N_{0}}{f\left(U \beta+y_{n}\right)} a Z_{n} \frac{\sin \left(\frac{z_{n} x}{L}\right) e^{y_{n} T_{1}-a(L-x)}}{y-y_{n}} \\
& +\frac{N_{2}-N_{1}}{f(U \beta+y) y} \cdot \frac{\left[(\beta-a-b)(a+b) e^{a(L+x)+b(x-L)}-(\beta-a+b)(a-b) e^{a(L+x)+b(L-x)}-2 e^{a x} b(-2 a+\beta) \cosh (b x)\right]}{2 e^{a L}((\beta-a) \sinh (b L)+b \cosh (b L))}
\end{aligned}
$$


Table 1. Aquifer Parameters Used in the Synthetic Example and for the Estimation of the Base Flow Rates for the Zwalm Catchment

\begin{tabular}{cccc}
\hline Parameter & Units & Synthetic Example & Zwalm \\
\hline$k$ & $\mathrm{~m} \mathrm{~s}^{-1}$ & 0.008 & 0.0002703 \\
$D$ & $\mathrm{~m}$ & 1.5 & 3.08 \\
$L$ & $\mathrm{~m}$ & 100 & 366 \\
$\theta$ & radians & 0.02 & 0.147 \\
$f$ & - & 0.34 & 0.07 \\
$\beta$ & $\mathrm{m}^{-1}$ & 0.005 & 0 \\
\hline
\end{tabular}

Inverse Laplace transform leads to

$$
\begin{aligned}
Q_{b}(t)= & A N_{1}+4 W_{0} K \sum_{n=1}^{\infty} \frac{N_{1}-N_{0}}{U \beta+y_{n}} Z_{n} \frac{z_{n}}{L} e^{y_{n} T_{1}-a L} e^{y_{n} t} \\
& +4 W_{0}\left(N_{2}-N_{1}\right) \sqrt{\frac{K t}{\pi}}
\end{aligned}
$$

$A$ is the catchment surface area $\left(\mathrm{m}^{2}\right)$, and is calculated as

$$
A=2 W_{0} \int_{0}^{L} e^{-\beta x}=2 W_{0} \frac{1-e^{-\beta L}}{\beta}
$$

Similarly as performed by Huyck et al. [2005], it is easy to show that in general (for a succession of $m$ recharge rates) the equation for the base flow for small values of time becomes

$$
\begin{aligned}
Q_{b}(t)= & A N_{m-1}+4 W_{0} K \sum_{n=1}^{\infty}\left[\sum_{i=1}^{m-1}\left(N_{i}-N_{i-1}\right) e^{y_{n} \mathcal{T}_{i}}\right] \\
& \cdot \frac{Z_{n}}{U \beta+y_{n}} \frac{z_{n}}{L} e^{y_{n} t-a L}+4 W_{0}\left(N_{m}-N_{m-1}\right) \sqrt{\frac{K t}{\pi}}
\end{aligned}
$$

\subsection{Further Simplification}

[22] Under the assumption that the recharge event prior to $N_{m}$ lasts very long, the double summation term in equation (27) will approximate zero, since $y_{n}$ is negative and $\mathcal{T}_{i}$ will become very large (equation (14)). Under this assumption the short-time expression becomes

$$
Q_{b}(t) \approx A N_{m-1}+4 W_{0}\left(N_{m}-N_{m-1}\right) \sqrt{\frac{K t}{\pi}}
$$

This situation can occur after a long rainless period. Thus if $N_{m-1}$ can be assumed to be equal to zero, this leads to the following expression:

$$
Q_{b}(t) \approx 4 W_{0} N_{m} \sqrt{\frac{K t}{\pi}}
$$

The first derivative of this expression with respect to time becomes

$$
\frac{d Q_{b}(t)}{d t} \approx 2 W_{0} N_{m} \sqrt{\frac{K}{\pi t}}
$$

Thus the relationship between $Q_{b}$ and $d Q_{b} / d t$ can be written as

$$
\frac{d Q_{b}}{d t} \approx 8 W_{0}^{2} N_{m}^{2} \frac{K}{\pi} Q_{b}^{-1}
$$

\subsection{Synthetic Example}

[23] In order to test the short-time expressions (equations (27) and (28)), a synthetic study was performed. An aquifer was subjected to a synthetic time series of recharge. Table 1 lists the aquifer parameters, and Table 2 lists the time series of recharge rates. The general solution (equation (13)), a numerical solution to the nonlinear equation (equation (4)), and the approximations (equations (27) and (28)) were analyzed, for a single aquifer of unit width at $x$ zero (thus $2 W_{0}$ in the equations is equal to one). The numerical solution to equation (4) has been obtained through a finite element discretization to solve the spatial derivatives, for which piecewise Lagrangian basis functions and piecewise uniform weight functions are used. The temporal derivative is solved using a Crank-Nicholson finite difference scheme. It should be noted that, when $N_{m-1}$ is zero, equations (28) and (29) will yield identical results.

[24] Figure 4 shows the results of this synthetic study. It can be seen that the linearized and nonlinear extended Boussinesq equations lead to relatively similar results. The peaks using the nonlinear equation are slightly higher, but for short times during the recharge events the outflows resulting from both equations are very similar. Further, in all cases equation (27) is, as expected, a good approximation of the exact solution for a short time during the recharge event. On the other hand, equation (28) is clearly not a good approximation for the decreasing limbs of the hydrograph. This can be explained by the omission of the double summation term, which cannot be neglected, in contrary to the assumptions made in the derivation of equation (28). However, for the increasing limbs of the hydrograph, a good match between the exact solution and equation (28) is obtained for short times during the recharge events. This good match is obtained even if the base flow before the onset of the peak is still relatively high, as for example occurs for the second peak. In other words, the base flow prior to the peak does not have to be zero in order for equation (28) or equation (29) to be valid.

[25] The results in Figure 4 thus indicate that equation (29) is a good approximation of the base flow for short times during the rising limbs of the base flow hydrographs, if they succeed a long rainless period. In section 4 , it will be

Table 2. Recharge Rates Used in the Synthetic Example

\begin{tabular}{lc}
\hline Recharge Rate $\left(\mathrm{mm} \mathrm{h}^{-1}\right)$ & Duration $(\mathrm{h})$ \\
\hline 1.2 & initial condition \\
5.28 & 20 \\
0 & 50 \\
8.088 & 20 \\
0 & 100 \\
4.56 & 10 \\
0 & 200 \\
13.92 & 10 \\
0 & 90
\end{tabular}




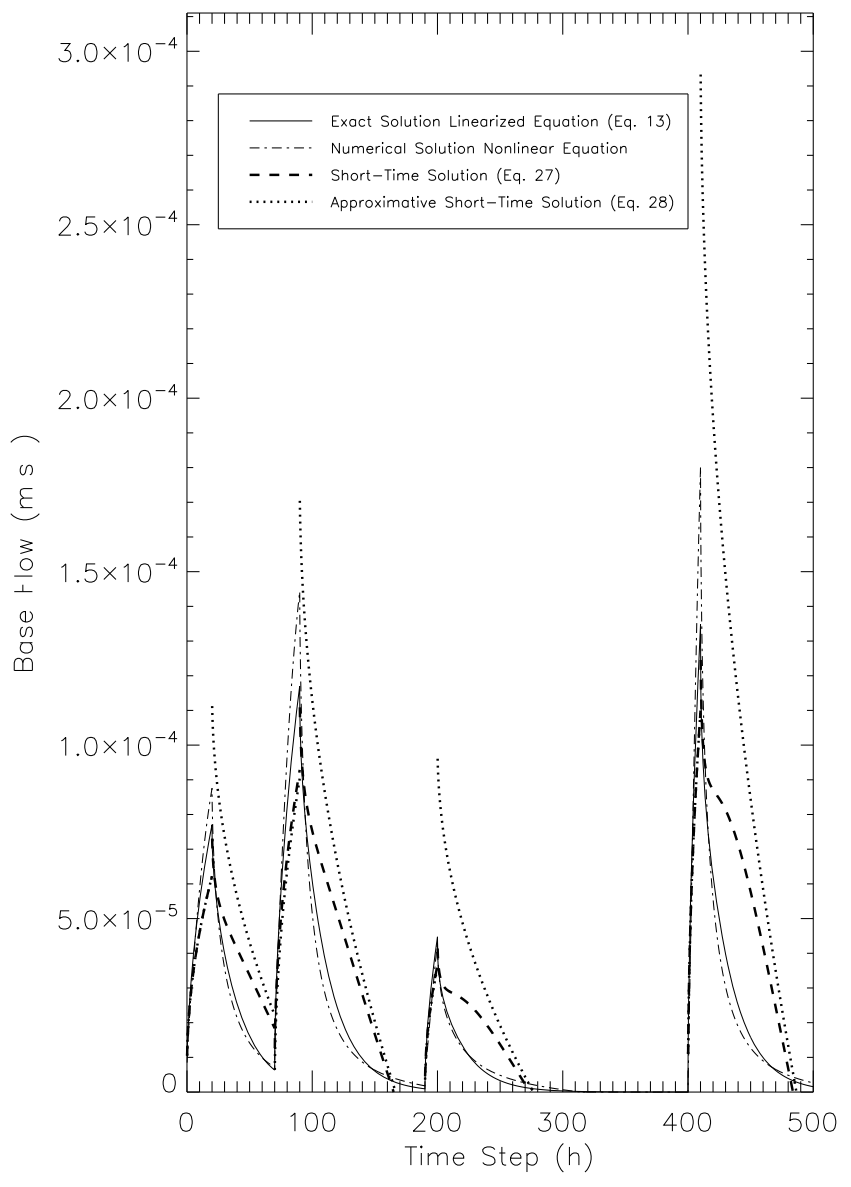

Figure 4. The results of the synthetic study. If the results of equation (28) are not visible, they coincide with the results of equation (27).

assessed whether this approximation can be used to estimate aquifer hydraulic parameters.

\section{Site and Data Description}

[26] The study has been performed in the Zwalm catchment in Belgium, of which Figure 5 shows the location. Troch et al. [1993] provide a complete description of this test site, only a short overview will be given here. The total drainage area of the catchment is $114.3 \mathrm{~km}^{2}$ and the total length of the perennial channels is $177 \mathrm{~km}$. The maximum elevation difference is $150 \mathrm{~m}$. The average annual temperature is $10^{\circ} \mathrm{C}$, with January the coldest month (mean temperature $3^{\circ} \mathrm{C}$ ) and July the warmest month (mean temperature $18^{\circ} \mathrm{C}$ ). The average annual rainfall is $775 \mathrm{~mm}$ and is distributed evenly throughout the year. The annual actual evapotranspiration is approximately $450 \mathrm{~mm}$.

[27] Meteorological forcing data with an hourly resolution (the model time step) from 1994 through 1998 were used in this study. The climatological station located in Kruishoutem, approximately $5 \mathrm{~km}$ outside the catchment, provided the precipitation needed by the models. Hourly discharge values at the outlet of the catchment were available for the entire study period.

[28] Application of a number of base flow filters for this catchment [Huyck et al., 2005] indicate that a very large fraction of the storm hydrographs consist of base flow, which makes this catchment very suitable for the analysis in this paper. Further, since we apply this method after a long dry period, it is likely that the initial rise will be strongly affected by subsurface flow that depends on the aquifer hydraulic properties that we want to estimate.

\section{Results}

\subsection{Model Validation}

[29] Table 1 lists the aquifer parameters that were used for the application of the model described in section 2. These parameters were determined by Pauwels et al. [2002]. The model was applied at an hourly time step from 1994 through 1998. The top panels of Figure 6 show the comparison of the modeled to the observed discharge for the simulation period, obtained using the linearized extended Boussinesq equation. The bottom panels of Figure 6 show this comparison, for the results obtained using the nonlinear extended Boussinesq equation. The results obtained using both equations are almost identical, indicating the validity of the linearized Boussinesq equation. The relatively low rootmean-square error (RMSE) and the regression line close to the $1: 1$ line indicate that the rainfall-runoff behavior of the catchment is adequately represented using this model, and that the resulting recharge rates can be deemed sufficiently realistic to be used in a synthetic experiment.

\subsection{Analysis of the Short-Time Expressions}

[30] In order to analyze the short-time expressions, equation (15) has been analyzed using the synthetically generated time series of base flow, obtained using the

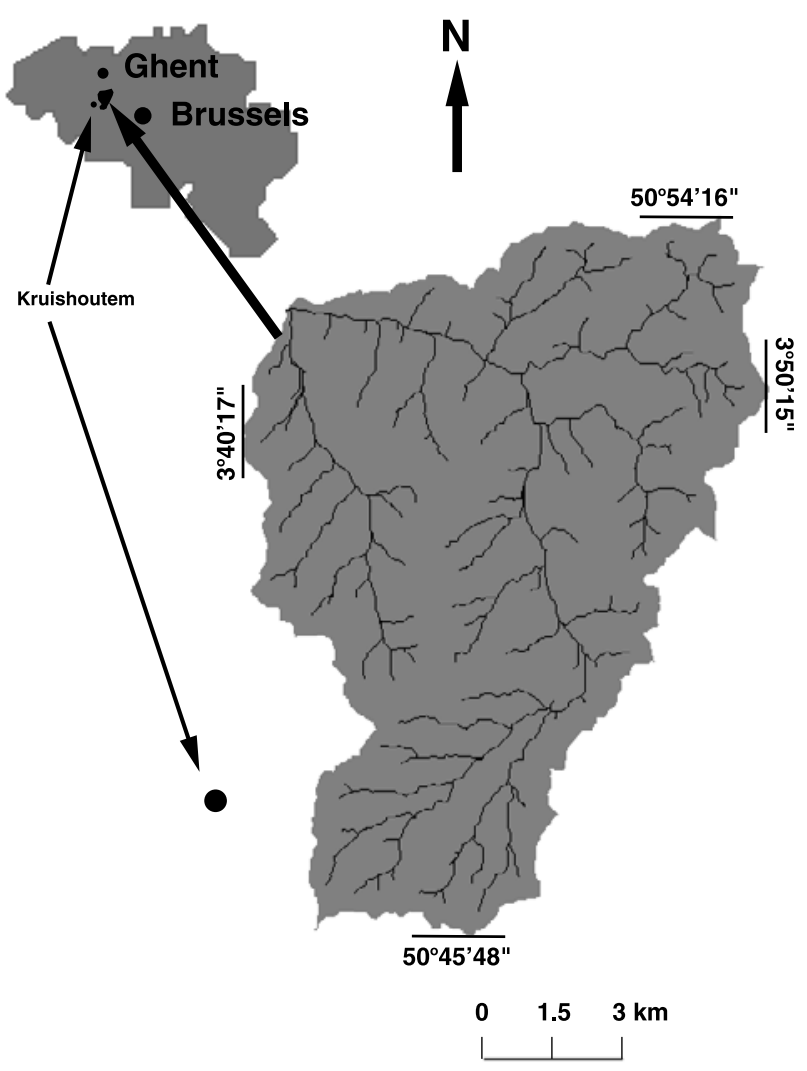

Figure 5. The location of the study site. 

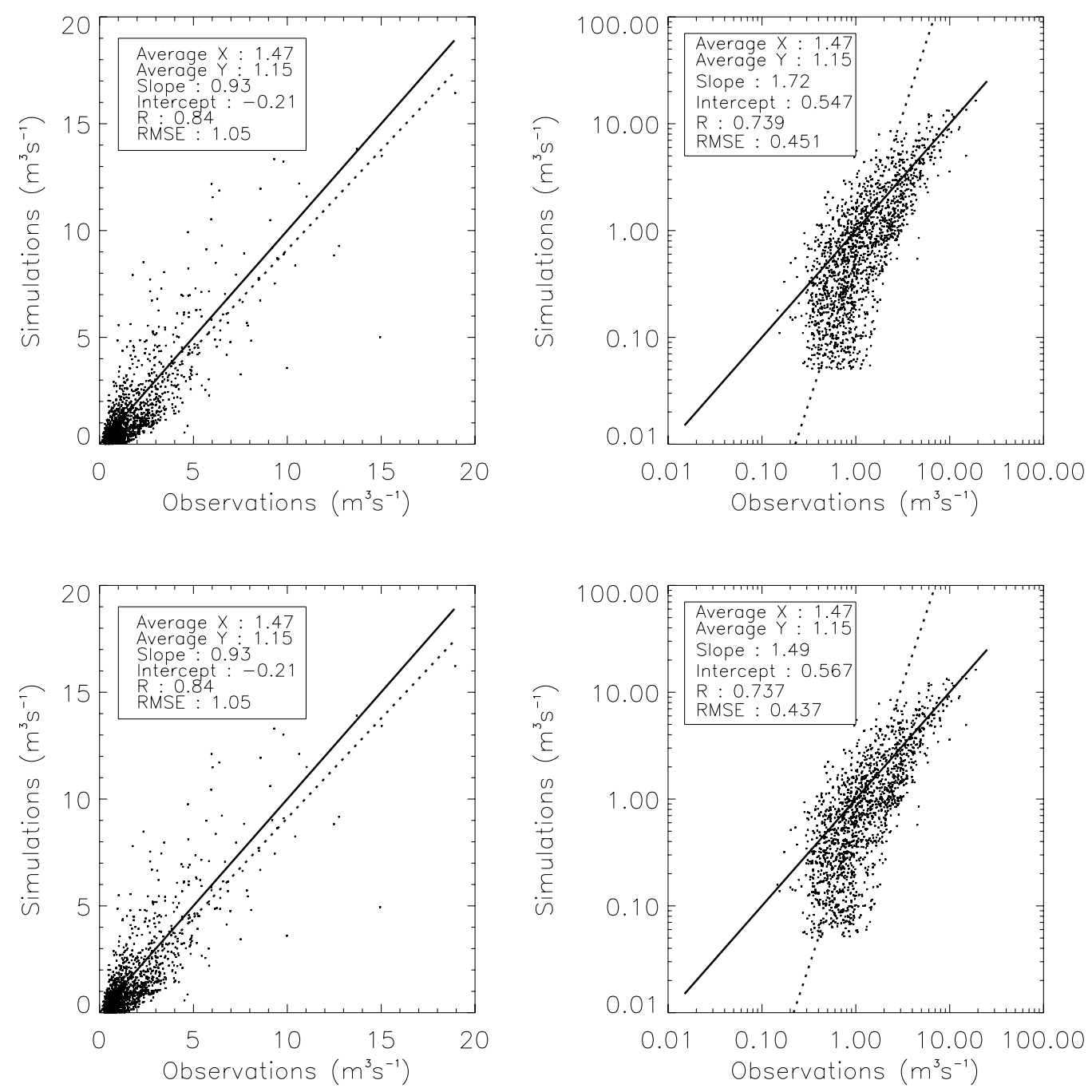

Figure 6. Validation of the modeled discharge for the Zwalm catchment showing (left) linear plots and (right) logarithmic plots. The solid line is the 1:1 line, and the dotted line is the regression line. (top) Results obtained using the linearized extended Boussinesq equation. (bottom) Results obtained using the nonlinear extended Boussinesq equation.

linearized extended Boussinesq equation. As stated in section 3.3, equation (15) has been derived under the assumption that the catchment was initially fully saturated. The results of the hydrologic model, as well as field observations, show that this situation does not occur at any time during the simulation period.

[31] The analysis has been performed as follows. For all modeled base flow values, the base flow and its first derivative with respect to time for the first time step in the decreasing limb of each hydrograph were retained, in order to approximate as accurately as possible the assumption of short times in the recession. The other time steps in the decreasing limbs were not used in the analysis. Under these conditions, even for relatively steep aquifers, the short-time solution to the Boussinesq equation is valid. Only data points for which no recharge occurred were used in the analysis.

[32] Figure 7 shows the logarithmic plot of the first derivative of the base flow with respect to time as a function of the base flow values for these time steps. Following equation (15), these data points should be located on a straight line with slope 3 . However, a linear regression through the data set yielded a regression line with slope 0.55 , intercept $5.89 \times 10^{-6} \mathrm{~m}^{1.65} \mathrm{~s}^{-1.45}$, and a correlation coefficient of 0.45 . The slope of 3 is thus not observed in this plot. This can be explained by the assumption of an initial full saturation in the derivation of equation (15), which does not occur in this catchment. It can thus be expected that this inconsistency between the theoretical assumptions in the derivation of equation (15) and the conditions in reality will lead to errors in the catchment hydraulic parameters. This will be assessed in section 5.3.

\subsection{Estimation of the Aquifer Hydraulic Parameters Through a Traditional Base Flow Recession Analysis}

[33] In this section, the modeled base flow values, obtained using the linearized extended Boussinesq equation, were used in a recession analysis. Figure 8 shows a logarithmic plot of the first derivatives of the modeled base flow values of the decreasing hydrograph limbs with respect to time as a function of the base flow values. Only data points for which no recharge occurs were used in the analysis. This plot was generated using the same time step 


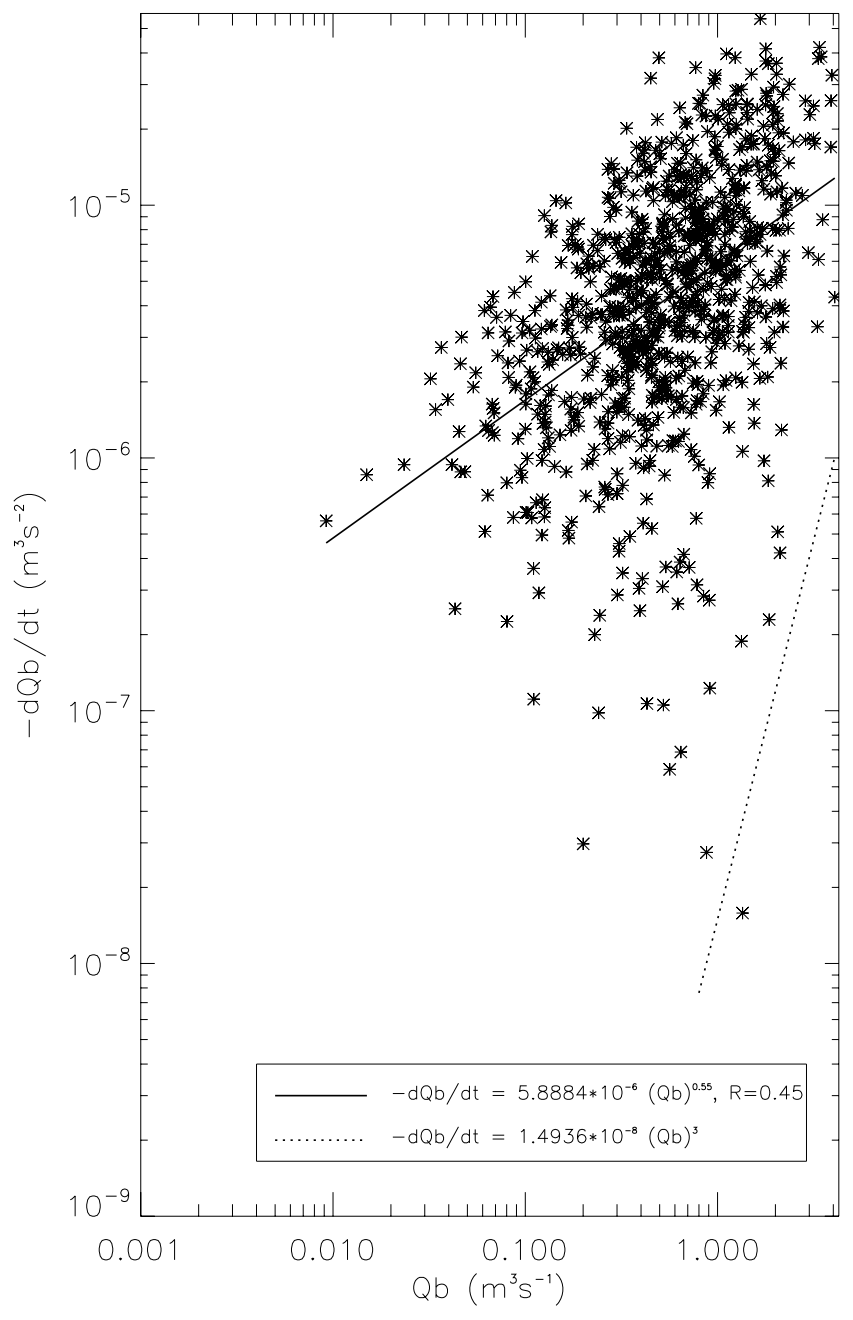

Figure 7. Analysis of the base flow recessions for short time.

at which the model was applied, more specifically $1 \mathrm{~h}$. Rupp and Selker [2006b] demonstrated that, if the streamflow data ara available with an imperfect precision and a certain level of noise, this approach can lead to misinterpretation of the recessions. They suggested the use of a variable time step in order to bypass this problem. However, in this paper, the base flow values were synthetically generated, and are thus perfectly known, without noise. In this study, as opposed to when real streamflow data are used, there is thus no benefit in using variable time steps in order to perform the recession analysis. It should be noted that the line and inverse $U$ shape of a number of data points for the lower values of $-d Q / d t$ can be explained by the effect of recharge during the recessions. This will cause similar discharge values to have different time derivatives. The inverse $\mathrm{U}$ shape can be explained by the relatively slow decrease of the base flow at the onset of a recession, the quicker decrease later in the recession, and the slower decrease toward the end of the recession. The aquifer hydraulic parameters $k$ and $D$ were determined using the intercepts of the straight lines with slope 1 and 3 in the $-d Q_{b} / d t$ versus $Q_{b}$ plots. Using these intercepts, and a value of 0.5 for the linearization constant $p$ (which was used to generate the base flow time series), equations (16) and (15) can be used to calculate $D$ and $k$.
[34] A number of interpretations of the location of these straight lines can be made. Traditionally, this type of recession analysis is performed on measured discharge values, not the base flow values themselves. For this reason, as described by Brutsaert and Nieber [1977] and Troch et al. [1993], low envelopes in the data sets are used, since the absolute value of the first derivative of surface runoff with respect to time is generally larger than this value for base flow. The use of these lower envelopes increases the chance that the intercepts are calculated on the base flow values, not on the discharge including surface runoff. Usually, the $5 \%$ or $10 \%$ lowest envelopes are used.

[35] However, in this section, the base flow values themselves are analyzed. Regarding the short-time solution with slope 3, the argument can be made that the value for $Q_{b}$ which is most likely to approximate the theoretically required

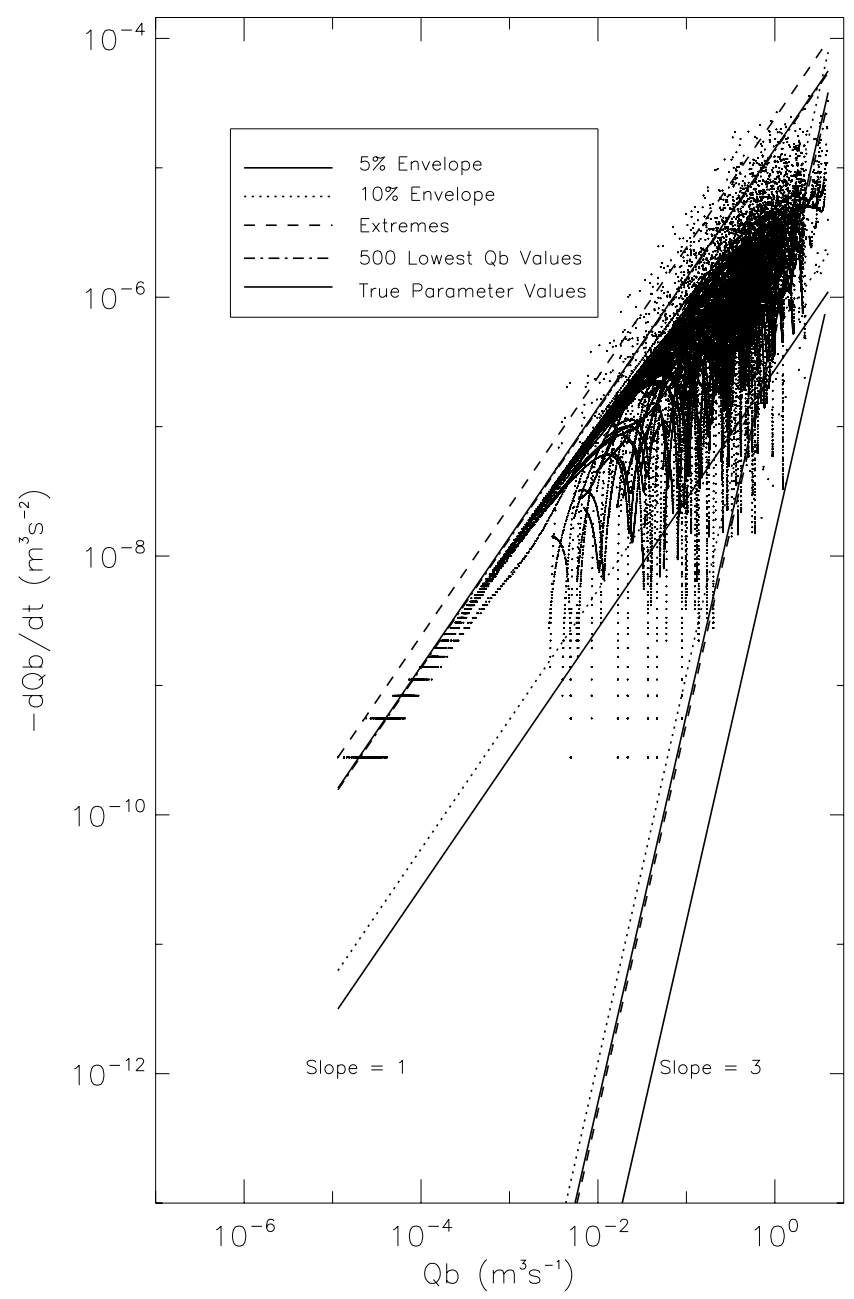

Figure 8. Calculation of the aquifer parameters through a traditional recession analysis. For reasons of completeness the $5 \%$ and $10 \%$ lower envelopes with a slope of 1 are also indicated on the plot, even though they are not used in the analysis. The dashed line is the line with slope 1 drawn through the lowest $Q_{b}$ value or the line with slope 3 drawn through the highest $Q_{b}$ value. The dash-dotted line is the linear regression with slope 1 drawn through the 500 lowest values for $Q_{b}$. The line with the 500 lowest values for $Q_{b}$ and the line with the true parameter values for slope 1 overlap. 


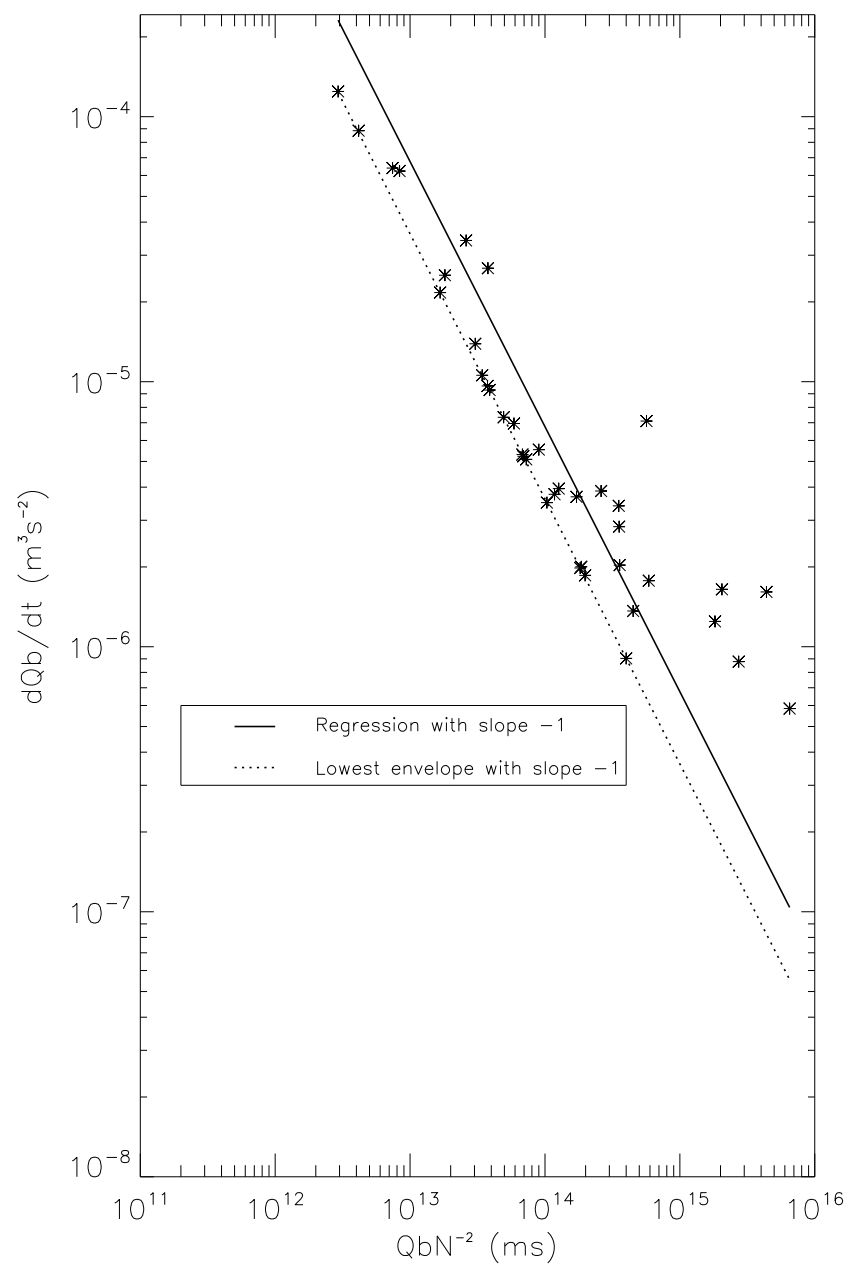

Figure 9. Analysis of the rising limbs of the base flow hydrographs, generated using the linearized extended Boussinesq equation, for short times after long rainless periods.

value (full saturation), is the largest value in the data set. Similarly, the value for $Q_{b}$ which is most likely to approximate the theoretically required value for the large time solution (slope 1), is the lowest value in the data set. For this reason, the intercept for the straight line with slope 3 was calculated using the highest obtained value for the base flow. Similarly, the intercept for the straight line with slope 1 was calculating using the lowest base flow value.

[36] The intercept for the straight line with slope 3 was found to be $5.11 \times 10^{-7} \mathrm{~s} \mathrm{~m}^{-6}$. For the straight line with slope 1 , this intercept was $2.42 \times 10^{-5} \mathrm{~s}^{-1}$. Using a drainable porosity of 0.07 , this corresponds to a value of $-1.50 \times$ $10^{-10} \mathrm{~m} \mathrm{~s}^{-1}$ for the saturated hydraulic conductivity, clearly an unrealistic value, and an aquifer depth $D$ of 8.57 , almost three times the correct value.

[37] Figure 8 shows that the calculated straight line with slope 1 approximates well the lower values for the discharge. In order to obtain a better estimate of the intercept of the straight line with slope 1 , a regression with slope 1 was drawn through the 500 lowest values of the discharge. This led to an intercept of $1.35 \times 10^{-5} \mathrm{~s}^{-1}$. Using the a priori known parameters for the catchment, a value of $1.398 \times$ $10^{-5} \mathrm{~s}^{-1}$ for $-y_{1}$ is obtained. The retrieved value thus approximates the true intercept value very well. Further, in order to take into account more data points in the calculation of the intercept for the straight line with slope 3 , the $5 \%$ and $10 \%$ lower envelopes were calculated. For the $5 \%$ lower envelope, the intercept is $5.96 \times 10^{-7} \mathrm{~s} \mathrm{~m}^{-6}$. It should be noted, for the $5 \%$ lower envelope with slope 3 , that this straight line is almost identical to the straight line drawn through the largest $Q_{b}$ value. These intercepts correspond to saturated hydraulic conductivity of $0.00015 \mathrm{~m} \mathrm{~s}^{-1}$ and an aquifer depth of $1.72 \mathrm{~m}$. For the $10 \%$ lower envelope this is $1.22 \times 10^{-6} \mathrm{~s} \mathrm{~m}^{-6}$, leading to a saturated hydraulic conductivity of $0.00013 \mathrm{~m} \mathrm{~s}^{-1}$ and an aquifer depth of $1.44 \mathrm{~m}$. As can be seen in Table 1, these values are quite different from the correct values. The argument can thus be made that, due to the inconsistencies between the conditions under which equation (15) has been applied and the assumptions used in its derivation, a base flow recession analysis may be able to only provide the correct order of magnitude of the aquifer hydraulic parameters. In section 5.4 a different methodology is proposed, leading to more accurate results.

\subsection{Application of the New Approximative Solution}

[38] In order to make the base flow analysis for short times consistent with the theoretical development, equation (31) was analyzed. As stated in section 3.6, this equation has been derived under the assumption that the recharge rate prior to the event is zero, and that the impact of the previous recharge rates can be neglected. This can occur after long rainless periods. For this reason, only the hydrographs succeeding a period with zero precipitation for at least 7 days were analyzed. A duration of 7 days was used as a compromise between the need to have enough data points to perform the analysis, and the use of rainless periods of sufficient length. For each rising limb in these base flow hydrographs, the first time step was retained, in order to approximate as well as possible the assumptions under which the short-time expression was derived. For these time steps, the first derivative of the base flow with respect to time was plotted as a function of the base flow divided by the square of the corresponding recharge rate, in a logarithmic plot. Figure 9 shows the result of this analysis. A linear regression through this data set yielded an intercept of $1.32 \times 10^{4} \mathrm{~m}^{4} \mathrm{~s}^{-1}$, a slope of -0.67 , and a correlation coefficient of -0.91 . The confidence intervals at the $95 \%$ level fully encompass the linear regression line with slope -1 drawn through the data set. This slope of -0.67 is thus statistically equal to the theoretical slope of -1 at the $95 \%$ confidence level. It should be stated that it is possible that for a number of data points in Figure 9 an important assumption used in the derivation of equation (31), more specifically no influence of previous recharge rates, is not valid. In section 5.6 a criterium is derived to eliminate these points from the analysis. In order to use this plot for the estimation of hydraulic parameters, two approaches can be used. On the one hand, a regression with slope -1 can be drawn through the data set. This regression line will thus take into account data points for which the theoretical requirements are not met, which can lead to errors in the estimated parameters. On the other hand, it can be argued that the points in the data set that best approximate the theoretical requirement of no influence of prior recharge rates on the base flow are the points with the lowest $d Q_{b} / d t$ for each value of $Q_{b} N^{-2}$. Therefore, a second straight line with slope -1 is drawn through the point with the lowest intercept in the data set, thus, in other words, through the 
point with the lowest $d Q_{b} / d t$ for the corresponding value of $Q_{b} N^{-2}$. As stated above, in section 5.6 a theoretically founded algorithm is described in order to determine the location of the straight line with slope -1 .

[39] Figure 9 shows the location of these two straight lines. It is important to note that many points in the data set are located almost exactly on the lower envelope. This indicates the validity of equation (31). The intercept of the regression with slope -1 is $6.76 \times 10^{8} \mathrm{~m}^{4} \mathrm{~s}^{-1}$, while for the lowest envelope this intercept is $3.61 \times 10^{8} \mathrm{~m}^{4} \mathrm{~s}^{-1}$. Equation (31) shows that this intercept is equal to $8 W_{0}^{2} K / \pi$. For the regression with slope -1 this corresponds to a $K$ of $1.08 \times 10^{-2} \mathrm{~m}^{2} \mathrm{~s}^{-3}$, while for the lower envelope this leads to a value of $5.79 \mathrm{~m}^{2} \mathrm{~s}^{-3}$ for $K$. Using equation (6) and the values in Table 1, it can be seen that the correct value for $K$ is equal to $5.88 \mathrm{~m}^{2} \mathrm{~s}^{-3}$. The value for $K$ obtained using the lower envelope is thus very similar to the correct value, indicating again the validity of equation (31).

[40] The hydraulic parameters $k$ and $p D$ (and consequently $D$ since $p$ is known) can be calculated using the plots in Figures 8 and 9. The intercept of the straight line with slope 1 in Figure 8, corresponding to the 500 lowest values of $Q_{b}$ (denoted as $I_{1}$ ), and the intercept of the lowest envelope in Figure 9 (denoted as $I_{2}$ ) can be used in the following system with two equations and two unknowns:

$$
\left\{\begin{array}{l}
I_{1}=\frac{k p D \cos \theta}{f}\left[\left(\frac{\beta p D \cos \theta-\sin \theta}{2 p D \cos \theta}\right)^{2}+\frac{z_{n}^{2}}{L^{2}}\right]+\frac{\beta k \sin \theta}{f} \\
I_{2}=\frac{8 W_{0}^{2} k p D \cos \theta}{f \pi}
\end{array}\right.
$$

These expressions have been obtained using equations (16), (14), and equation (6) for $I_{1}$, and equations (31) and (6) for $I_{2}$. It should be noted that these are limit cases, so an infinite summation is no longer necessary. Using the values for $I_{1}$ and $I_{2}$ that were explained above, a value of $3.08 \mathrm{~m}$ for $D$ and $0.000266 \mathrm{~m} \mathrm{~s}^{-1}$ for $k$ were obtained. Table 1 shows that these are relatively close to the original parameter values.

\subsection{Physical Interpretation of the New Methodology}

[41] To summarize, the new method uses two equations that are valid for relatively dry aquifers. The first expression (for large values of time) has been derived under the assumption that the aquifer has been draining for a long time. The second expression (for short times) has been derived for the case of replenishing of the aquifer after a long rainless period.

[42] Following the discussion in section 3.2, in both cases the value for $p$ should be small. However, in order to generate the base flow time series used in the analysis, a constant value of 0.5 for $p$ was used. Logically, the correct value for $p D$, and consequently $D$, was retrieved, since this value was used to generate the time series of base flow. In this context, it should be noted that the synthetic example was used to demonstrate the correctness of the long- and short-time equations. However, if the new methodology were applied on observed base flow values, a value for $p D$ consistent with a low value for $p$ would be retrieved, since the aquifer is relatively dry after a long recession and at the onset of a replenishing after a long rainless period. The value for $D$ can thus be expected to be an order of magnitude larger than the retrieved value for $p D$. In any case, it is important to note that both the short-time and the long-time solutions, used to calculate $p D$, are both valid for relatively dry aquifers, and consequently for low values of $p$. Since the water levels in the aquifer are low when the methodology is applied, it can be assumed that the hydraulic conductivity values are invariant with depth throughout the water table profile. The new method can thus be expected to provide good estimates of the value of the hydraulic conductivity of the lower part of the aquifer.

[43] However, if a traditional base flow recession analysis is performed, the equations for long and short times are valid for different values for $p$. The short-time solution has been derived for an initially saturated aquifer, and consequently a value for $p$ that approximates one. On the other hand, the long-time solution has been derived for an aquifer that has been draining freely for a long time, and thus a relatively low value for $p$. However, in the estimation of the aquifer parameters using these expressions, a constant value for $p$ (or $p D$ ) needs to be used. It can thus be stated that the newly developed methodology is more consistent with its theoretical requirements than a traditional base flow recession analysis.

\subsection{Selection of Analysis Data Points}

[44] A remaining issue is the selection of the appropriate points in Figure 9 to perform the analysis. As stated above, the straight line with slope -1 can be drawn through the data point with the lowest value for the intercept. However, a more appropriate manner to select these points is the following.

[45] Equations (29) and (30) show that, for the points for which these equations are valid, the following relationship is true:

$$
\frac{Q_{b}(t)}{\frac{d Q_{b}(t)}{d t}} \approx 2 t
$$

Thus, if data points are sampled at a regular interval $\triangle t(\mathrm{~s})$, and if the assumptions leading to equations (29) and (30) are valid, a division of the discharge by the first derivative of the discharge with respect to time is approximately equal to twice the data time step. Only base flow values and their first time derivatives are thus needed to select the analysis data points.

[46] This criterium can be used to select the analysis data points in Figure 9. All data points for which this division was between $1.95 \Delta t$ and $2.05 \triangle t$ were retained for the analysis. Indeed, a linear regression through the remaining data points (14 out of the original 38) yielded a slope of -1 , an intercept of $3.78 \times 10^{8} \mathrm{~m}^{4} \mathrm{~s}^{-1}$, and a correlation coefficient of -1 . The location of this straight line is thus practically identical to the location of the lower envelope in Figure 9. Application of this intercept to equation (32) resulted in an aquifer depth $D$ of $3.15 \mathrm{~m}$ and a saturated hydraulic conductivity $k$ of $0.0002718 \mathrm{~m} \mathrm{~s}^{-1}$. Table 1 shows that these values are again very close to the true parameters.

\section{Dimensional Analysis}

\subsection{Introduction to the Analysis}

[47] An important aspect in the application of a differential equation, or in general in the application of any model, 
is an understanding of the sensitivity of the results to the different parameter values. In this section, it is first assessed analytically whether the outflow resulting from the linearized extended Boussinesq equation (equation (8)) is sensitive to the three aquifer hydraulic parameters $(f, p D$, and $k)$. Since these parameters are determined using analytical solutions to equation (8) for short and large times, it is also investigated whether this sensitivity is reflected in the resulting parameter values. In other words, it is checked whether the parameter sensitivity of the short- and largetime solutions is consistent with the parameter sensitivity of the governing differential equation (equation (8)).

\subsection{Analysis of the Governing Equation}

[48] Using the Darcy equation (equation (7)), the first derivative of the base flow with respect to time can be written as

$$
\frac{d q}{d t}=-f K \frac{\partial}{\partial x}\left(\frac{\partial h}{\partial t}\right)-f U \frac{\partial h}{\partial t}
$$

Substitution of equation (8) leads to

$$
\begin{aligned}
\frac{d q}{d t}= & -f K^{2} \frac{\partial^{3} h}{\partial x^{3}}-f K(U-\beta K) \frac{\partial^{2} h}{\partial x^{2}}+f U K \beta \frac{\partial h}{\partial x}-f U K \frac{\partial^{2} h}{\partial x^{2}} \\
& -f U(U-\beta K) \frac{\partial h}{\partial x}+f U^{2} \beta h-f U \frac{N}{f}
\end{aligned}
$$

Rearrangement results in the following expression:

$$
\begin{aligned}
\frac{d q}{d t}= & -f K^{2} \frac{\partial^{3} h}{\partial x^{3}}-f K U \frac{\partial^{2} h}{\partial x^{2}}+f K^{2} \beta \frac{\partial^{2} h}{\partial x^{2}}+f U K \beta \frac{\partial h}{\partial x}-f U K \frac{\partial^{2} h}{\partial x^{2}} \\
& -f U^{2} \frac{\partial h}{\partial x}+f U K \beta \frac{\partial h}{\partial x}+f U^{2} \beta h-U N
\end{aligned}
$$

A number of terms can then be regrouped:

$$
\begin{aligned}
\frac{d q}{d t}= & -K \frac{\partial^{2}}{\partial x^{2}}\left(f K \frac{\partial h}{\partial x}+f U h\right)+K \beta \frac{\partial}{\partial x}\left(f K \frac{\partial h}{\partial x}+f U h\right) \\
& -U \frac{\partial}{\partial x}\left(f K \frac{\partial h}{\partial x}+f U h\right)+U \beta\left(f K \frac{\partial h}{\partial x}+f U h\right)-U N
\end{aligned}
$$

Substituting the Darcy equation (equation (7)), this equation can be further simplified. Rearrangement finally leads to

$$
\frac{d q}{d t}=K \frac{\partial^{2} q}{\partial x^{2}}+(U-K \beta) \frac{\partial q}{\partial x}-U \beta q-U N
$$

Equation (6) shows that, regarding the hydraulic parameters, both $K$ and $U$ are dependent on $k / f$, and that $K$ is further dependent on $p D$. $f$ does no longer appear as a separate parameter in equation (38). One would thus expect the analytical expression for the base flow (equation (13)) to be dependent on $k / f$ and $p D$ (thus in fact on two parameters), not on the three parameters separately. In section 6.3 it will be assessed whether or not this is the case.

\subsection{Analysis of the Analytical Solution}

[49] For the purpose of a dimensional analysis, equation (13) is rewritten, substituting the expression for $y_{n}$ from equation (14):

$$
\begin{aligned}
Q_{b}(t)= & A N_{m}-\left(N_{m}-N_{m-1}\right)\left[\sum_{n=1}^{\infty} \frac{4 W_{0} Z_{n}}{a^{2}+\frac{z_{n}^{2}}{L^{2}}} \frac{z_{n}}{L} e^{y_{n} t-a L}\right] \\
& -\sum_{i=1}^{m-1}\left(N_{i}-N_{i-1}\right)\left[\sum_{n=1}^{\infty} \frac{4 W_{0} Z_{n}}{a^{2}+\frac{z_{n}^{2}}{L^{2}}} \frac{z_{n}}{L} e^{y_{n}\left(t+T_{i}\right)-a L}\right]
\end{aligned}
$$

With respect to the hydraulic parameters, this expression is dependent on $a, z_{n}, Z_{n}$, and $y_{n}$. $a$ can be rewritten as (equation (14))

$$
a=-\frac{U}{2 K}+\frac{\beta}{2}=-\frac{k \sin \theta}{2 k p D \cos \theta}+\frac{\beta}{2}=-\frac{\tan \theta}{2 p D}+\frac{\beta}{2}
$$

This shows that $a$, with respect to the hydraulic parameters, is only dependent on the effective aquifer thickness $p D$. Equation (14) then shows that both $z_{n}$ and $Z_{n}$ are independent of $f$ and $k$, and will depend only on $p D$. Thus, for all the terms inside the summation for $n$, only $y_{n}$ is dependent on $k$ and $f . y_{n}$ can be rewritten as (equation (14))

$$
y_{n}=-U\left[\frac{K}{U}\left(a^{2}+\frac{z_{n}^{2}}{L^{2}}\right)+\beta\right]=-\frac{k}{f} \sin \theta\left[\frac{\tan \theta}{p D}\left(a^{2}+\frac{z_{n}^{2}}{L^{2}}\right)+\beta\right]
$$

This means that, with respect to the hydraulic parameters, $y_{n}$ is dependent only on $k / f$ and $p D$, while $Z_{n}, z_{n}$, and $a$ are dependent only on $p D$. The results in this section imply that the base flow modeled using the linearized Boussinesq equation will depend only on $k / f$ and $p D$, consistent with the reasoning in section 6.2. Application of the metahillslope model (meaning the hydrologic model in which the catchment is represented by two aquifers draining into the stream network) with the values for $f$ and $k$ multiplied by two confirmed this conclusion.

[50] This finding has a number of important consequences. First, in order to estimate the parameters through a base flow analysis, using the algorithm outlined in section 5.4, the same value for $p D$ (and consequently $D$ ) should be retrieved, regardless of the value for $f$. Further, whatever the value for $f$ used in the analysis, the obtained value for $k / f$ should be constant. In other words, the use of a traditional base flow recession analysis [Brutsaert and Nieber, 1977; Troch et al., 1993; Huyck et al., 2005] results in a system with two equations and three unknowns. In this case, the system reduces to two equations and two unknowns, implying, as stated above, that $k / f$ and $p D$ can be determined uniquely using the intercepts in equation (32). In sections 6.4 and 6.5 it will be investigated whether or not this is the case. Second, if the parameters need to be retrieved for the purpose of using them to model base flow using the linearized Boussinesq equation, retrieving the parameters $k / f$ and $p D$ is sufficient. Thus, the value for $f$ is irrelevant for this purpose. However, if the water table profile needs to be modeled, a reasonable 


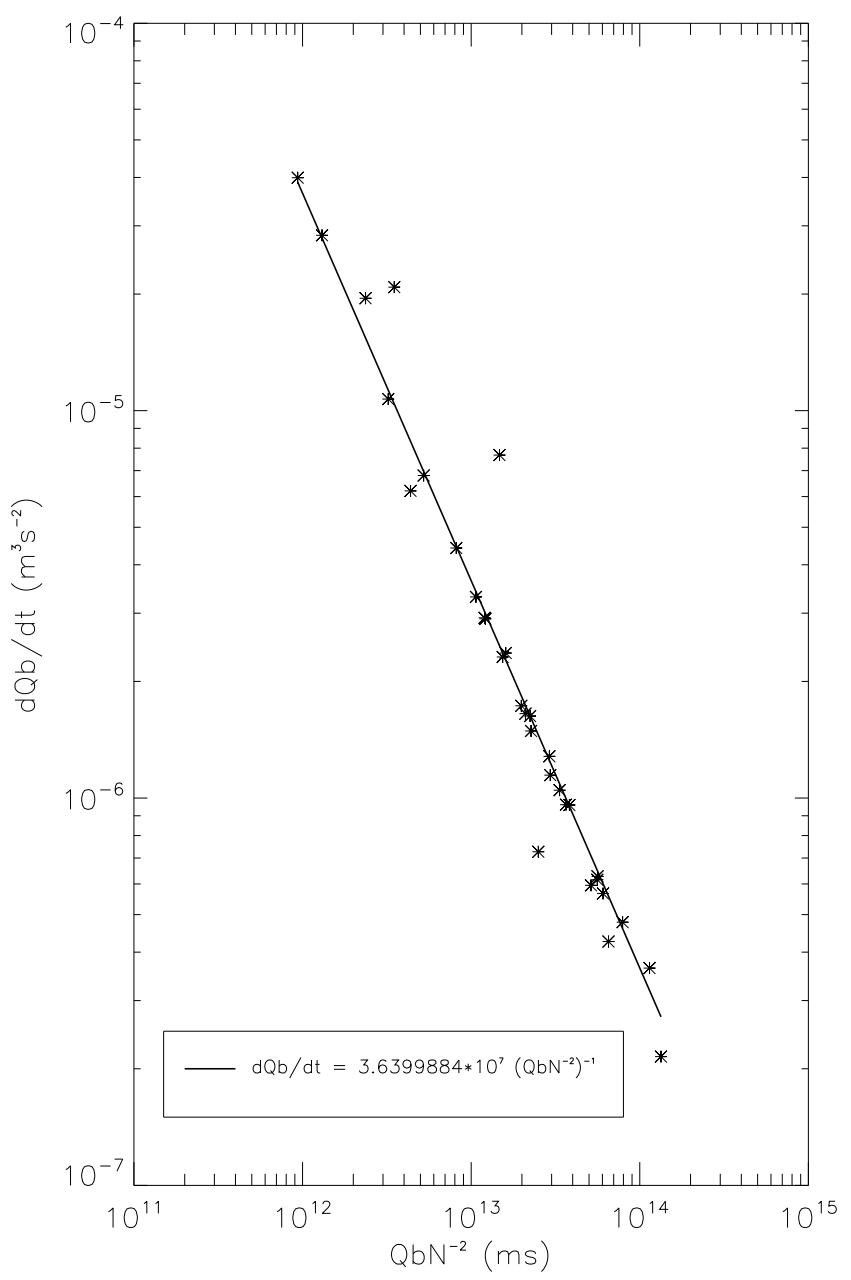

Figure 10. Analysis of the rising limbs of the base flow hydrographs, generated using the nonlinear extended Boussinesq equation, for short times after long rainless periods.

estimate for $f$ remains necessary. Examples of values for this parameter can be found in the work by Bear [1972].

\subsection{Analysis of the Approximations for Short Time}

[51] Troch et al. [1993] argue that, of the three parameters that need to be estimated $(D, k$ and $f)$, the drainable porosity $f$ is the least scale-dependent parameter. For this reason, Troch et al. [1993] use field estimates for this parameter, obtained using pumping well tests, for their analysis. The results of Troch et al. [1993], confirmed by the findings of Huyck et al. [2005], demonstrate that the use of different values for $f$, even if they are not strongly different, has a direct impact on the retrieved parameter values for $k$ and $D$. The results from both studies further clearly show that $k / f$ is not independent of the value used for the drainable porosity. In this context it should be noted that the results of Troch et al. [1993] are obtained using the nonlinear Boussinesq equation, while the results of Huyck et al. [2005] are obtained using the linearized version, more specifically equation (8). Thus, the methodology of Troch et al. [1993] correctly leads to an underdefined system of two equations and three unknowns, and variable estimates of $D$ and $k / f$ as a function of $f$, but the results of Huyck et al. [2005] are clearly not consistent with the above reasoning. This latter discrepancy can only be explained by the use of unrealistic initial conditions in the derivation of the short-time expression, which was derived using initial full saturation and zero recharge.

[52] A closer look at equation (32) shows that $I_{2}$ is dependent on $k / f$ and $p D$. The same statement can be made regarding $I_{1}$. Thus, the short-time expressions derived in this paper are consistent with the theory that the base flow, with respect to the aquifer hydraulic parameters, is only dependent on $k / f$ and $p D$. In section 6.5 , it will be verified whether the use of a different value for $f$ will lead to consistent estimates of $k / f$ and $p D$ (and consequently $D$ ) or not.

\subsection{Application to the Synthetic Data}

[53] In order to test the hypothesis that the estimates of $k / f$ and $D$ are independent of the value for $f$, the aquifer parameters calculated in section 5.4 were recalculated with values of 0.035 and 0.14 for $f$ (the original value divided by and multiplied by 2). For $f$ equal to 0.035 , the obtained value for $k$ was equal to $0.000133 \mathrm{~m} \mathrm{~s}^{-1}$, while $D$ was equal to $3.08 \mathrm{~m}$. On the other hand, for $f$ equal to 0.14 , the values for $k$ and $f$ were $0.000532 \mathrm{~m} \mathrm{~s}^{-1}$ and $3.08 \mathrm{~m}$, respectively. $k / f$ and $D$ are thus constant in all three cases. The results in this section thus indicate that the relationships between $d Q_{b} / d t$ and $Q_{b}$, derived for short and long times, and the resulting parameter estimates, are consistent with the governing equation.

\section{Application to the Nonlinear Equation}

[54] As shown in section 3.7, the linearized and nonlinear extended Boussinesq equations behave relatively similarly for the rising limbs in the base flow hydrographs after long rainless periods. It can thus be expected that equation (31) will also be valid if base flow values are generated using the nonlinear equation.

[55] Figure 10 shows the relationship between $Q_{b} N^{-2}$ and $d Q_{b} / d t$, generated using the nonlinear extended Boussinesq equation, for the same time steps as are shown in Figure 9, thus immediately after at least one week without precipitation. A linear regression through the data set yielded an intercept of $1.82 \times 10^{8} \mathrm{~m}^{4} \mathrm{~s}^{-1}$, a slope of -1.0529 , and a correlation coefficient of -0.98 . These results (the slope very close to -1 ) indicate the applicability of the newly derived short-time solution for the estimation of aquifer lower layer hydraulic conductivity values for the nonlinear extended Boussinesq equation.

[56] Similar as in section 5.6, a linear regression was drawn through the points for which the division of $Q_{b}$ by $d Q_{b} / d t$ was between $1.95 \triangle t$ and $2.05 \triangle t$. This yielded an intercept of $3.64 \times 10^{7} \mathrm{~m}^{4} \mathrm{~s}^{-1}$, a slope of -1 , and a correlation coefficient of -1 . This is further proof that the new short-time solution can be used to estimate aquifer hydraulic parameters if the nonlinear extended Boussinesq equation is used.

[57] The major difference between the use of the nonlinear equation as compared to the linearized equation, is the fact that the slope of 1 in the relationship between the base flow and the first time derivative thereof for large values of time (a consequence of the linearization of the governing equation) will not be retrieved (for a horizontal aquifer this slope should theoretically be 1.5) [Rupp and Selker, 2006a]. 
Table 3. Calculation of $k / f$ Using the Intercept of the Plot With Slope $-1^{\text {a }}$

\begin{tabular}{lcc}
\hline$p D(\mathrm{~m})$ & $p$ & $k / f\left(\mathrm{~m} \mathrm{~s}^{-1}\right)$ \\
\hline 0.02 & 0.00649 & 0.023061 \\
0.04 & 0.01299 & 0.011531 \\
0.06 & 0.01948 & 0.007687 \\
0.08 & 0.02597 & 0.005765 \\
0.1 & 0.03247 & 0.004612 \\
0.12 & 0.03896 & 0.003844 \\
0.14 & 0.04545 & 0.003295 \\
0.16 & 0.05195 & 0.002883 \\
0.18 & 0.05844 & 0.002562 \\
0.2 & 0.06494 & 0.002306 \\
\hline
\end{tabular}

${ }^{\mathrm{a}}$ The calculation is generated using the nonlinear extended Boussinesq equation. The true $k / f$ is $0.003861 \mathrm{~m} \mathrm{~s}^{-1}$.

As a consequence, equation (16) cannot be used in the retrieval of the aquifer hydraulic parameters. Equation (31) contains three unknowns, more specifically $p D, k$, and $f$. Consistent with the explanation in section 3.2, if low values of $p$ are used, the correct order of magnitude of $k / f$ should be retrieved. In order to assess this hypothesis, the value for $k / f$ obtained using the intercept of $3.64 \times 10^{7} \mathrm{~m}^{4} \mathrm{~s}^{-1}$ was calculated for different values of $p D$. Table 3 shows the results of this computation. Indeed, for a value of $p$ of approximately 0.03 , the correct value of $k / f$ is retrieved.

[58] The important consequence of this analysis is that, if base flow is modeled using the nonlinear extended Boussinesq equation, the order of magnitude of the aquifer lower layer hydraulic conductivity can be estimated using only one equation. A low value of $p D$ should be used, and an estimate of $f$ is needed in that case.

\section{Discussion and Conclusions}

[59] The objective of this paper was to develop a methodology for the estimation of catchment-scale aquifer lower layer hydraulic conductivity values, consistent with the conditions under which the required equations have been derived. First, the applicability of a traditional base flow recession analysis has been examined. The assumptions under which the expressions for small values of time have been derived, an initially fully saturated aquifer not subject to recharge, are in most cases not realistic. It has been shown that the use of this type of relationship does provide a good estimate of the order of magnitude of the catchmentaveraged saturated hydraulic conductivity, but that the obtained values are prone to a large uncertainty.

[60] A different expression for the base flow has been derived, valid for short times during a recharge event. The equation is valid for the rising limb of a hydrograph after a long rainless period. Based on this expression, an inverse relationship between the base flow and its first derivative with respect to time has been obtained. Apart from the aquifer hydraulic parameters, this relationship also depends on the recharge rate. A synthetic experiment has shown that, using this new relationship, combined with the relationship for large times for base flow recessions, accurate estimates of the catchment hydraulic parameters for the lower layers of the aquifer are obtained.

[61] It has also been shown analytically that the base flow, modeled using the linearized Boussinesq equation, is dependent only on $k / f$ and $p D$, not on the three parameters $(k, f$, and $p D)$ separately. This finding has been confirmed through a numerical experiment. This leads to the important implication that knowledge on the values of $k / f$ and $p D$ is sufficient if the base flow needs to be modeled using the linearized Boussinesq equation. Estimates of the drainable porosity are no longer needed for this purpose. Using the newly developed expression for the base flow for short times, the estimates of, on the one hand, the aquifer transmissivity $p D$, and, on the other hand, the ratio $k / f$, have been found to be insensitive to the drainable porosity used in the analysis. This is consistent with the theory, and more robust parameters are thus obtained than in the case of a traditional recession analysis. However, if water table profiles need to be modeled, reasonable estimates for $f$ remain necessary.

[62] If base flow is modeled using the nonlinear extended Boussinesq equation, it has been shown that the newly derived expression can be used without a second equation for large values of time in order to estimate the order of magnitude of the aquifer hydraulic conductivity. $p D$ needs to be assumed to be small for this purpose, and an estimate of the aquifer drainable porosity is needed. Regarding the choice between the linearized and nonlinear Boussinesq equations, a strong debate is still ongoing about the conditions under which the two versions can be applied. An analysis of the applicability of both versions has been performed by Harman and Sivapalan [2009b].

[63] Even though the new expression is clearly accurate, an operational application suffers from two drawbacks. First, the analysis needs to be performed on base flow values, not on total discharge. Empirical digital base flow filters [Lyne and Hollick, 1979; Boughton, 1993; Chapman and Maxwell, 1996; Jakeman and Hornberger, 1993] should not be used to obtain these base flow values, since these algorithms are usually constructed quite simplistically, which will lead to a nonnegligible uncertainty in the base flow estimates. In other words, the shape of the rising limb is largely an artifact of the filter used. This will have an impact on the intercepts of the straight lines with slope -1 and 1 in the logarithmic plots, and consequently on the obtained parameters. Physically based base flow filters [Furey and Gupta, 2001, 2003; Huyck et al., 2005] should not be used either, since these require catchment-averaged parameters, which need to be estimated through a base flow analysis. It is therefore recommended to apply the equations on direct estimates of base flow. Recently, significant advances have been made on the use of isotopes [Tetzlaff et al., 2007; Nakaya et al., 2007; Tetzlaff and Soulsby, 2008; Liu et al., 2008; Lyon et al., 2008; Desilets et al., 2008] or distributed temperature sensing [Westhoff et al., 2007] for the analysis of hydrograph composition. It should thus be possible to obtain a long enough time series of base flow to perform the analysis. A more difficult problem is the quantification of the catchment-averaged recharge during the rising limb of the hydrograph. Since the recharge appears to the power two in equation (31), it must be determined with a relatively high accuracy. Recent results of a combination of soil moisture profile observations and gravity data indicate that changes in the amount of storage in the groundwater layer can be related to changes in detected gravity [Swenson et al., 2008]. If base flow rates are obtained using one of the 
previously mentioned methods, the application of this methodology at finer spatial and temporal scales using terrestrial gravity measurements [Hasan et al., 2008], combined with a simple mass balance of the saturated soil zone, could result in recharge rates that are accurate enough to perform the rising limb analysis.

[64] The results in this paper also reinvigorate the discussion on the appropriateness of linearized versions of the Boussinesq equation for the purpose of modeling the base flow in hydrologic models, as opposed to using the nonlinear equation. Rupp and Selker [2006a] provide an overview of the disadvantages of using the linearized equation. However, the question can be raised whether it is better to use an approximative equation, with accurate parameter estimates, or a more correct equation, with relatively erroneous parameter estimates.

[65] The overall conclusion from this paper is that, if one is interested in knowing catchment-averaged hydraulic parameters, a traditional base flow recession analysis will lead to results that will give an indication of the order of magnitude of these parameters. However, if more accurate estimates are needed, the effect of unrealistic initial conditions and recharge rates cannot be neglected. In this case, the rising limb of the base flow hydrographs can be used to estimate the catchment-averaged hydraulic conductivity values, which is the important conclusion from this paper. In this case, the application of the methodology developed in this paper could be considered.

\section{Appendix A: Solution of the Partial Differential Equation}

\section{A1. Solution for an Initially Saturated Aquifer}

[66] Equation (8) was solved by Huyck et al. [2005] for temporally variable recharge rates. Since the derivation of the new short-time expression is based on the equations of Huyck et al. [2005], this solution is repeated here.

[67] Laplace transformation of equation (8) leads to the following Ordinary Differential equation (ODE) with the initial condition $h=D$ and under a constant recharge rate $N_{0}$ :

$$
K \frac{d^{2} F}{d x^{2}}+(U-K \beta) \frac{d F}{d x}-(U \beta+y) F=-D-\frac{N_{0}}{f y}
$$

where $F$ denotes the Laplace transform of $h$ and $y$ is the Laplace variable. The solution to this ODE is

$$
\begin{aligned}
F= & \left(\frac{D}{U \beta+y}+\frac{N_{0}}{f(U \beta+y) y}\right) \\
& \cdot \frac{(\beta-a-b) e^{a(L+x)+b(x-L)}-(\beta-a+b) e^{a(L+x)+b(L-x)}-2 e^{a x}(-2 a+\beta) \sinh (b x)}{2 e^{a L}((\beta-a) \sinh (b L)+b \cosh (b L))} \\
& +\frac{D}{U \beta+y}+\frac{N_{0}}{f(U \beta+y) y}
\end{aligned}
$$

$$
y_{n}=K\left(-a^{2}-\frac{z_{n}^{2}}{L^{2}}\right)-U \beta
$$

We introduce the following notation:

$$
Z_{n}=\frac{z_{n}\left[e^{a L}+\frac{2 a-\beta}{\beta-a} \cos \left(z_{n}\right)\right]}{L^{2}(\beta-a)^{2}+L(\beta-a)+z_{n}^{2}}
$$

where $a=-\frac{U-K \beta}{2 K}\left(\mathrm{~m}^{-1}\right)$ and $b=\sqrt{a^{2}+\frac{U \beta+y}{K}}\left(\mathrm{~m}^{-1}\right)$.

[68] The inverse Laplace transform can be written as the sum of the residues at each pole: $h(x, t)=\Sigma R_{i}$. For the poles of the first order, these residues are

$$
R_{i}=\lim _{y \rightarrow y_{i}}\left[\left(y-y_{i}\right) F(y) e^{y t}\right]
$$

Since $y_{n}$ is negative, the sum in equation (A7) becomes zero when $t \rightarrow \infty$. The steady state water table profile is thus given by

$$
h_{0}\left(x, N_{0}\right)=\frac{N_{0}}{f U \beta} \cdot\left[1-e^{(2 a-\beta) x}+\frac{-e^{a x}(-2 a+\beta) \sinh (x(\beta-a))}{(\beta-a) e^{\beta L}}\right]
$$


The expression for $F$ in the Laplace domain is

$$
\begin{aligned}
F= & \frac{U \beta N_{2}+N_{1} y}{y f U \beta(U \beta+y)}+N_{1} \frac{(2 a-\beta)}{2 y f U \beta(\beta-a) e^{\beta L}} e^{\beta x}-N_{1} \frac{2(\beta-a) e^{\beta L}+(2 a-\beta)}{2 y f U \beta(\beta-a) e^{\beta L}} e^{(2 a-\beta) x} \\
& +2 \sum_{n=1}^{\infty} \frac{N_{1}-N_{0}}{f\left(U \beta+y_{n}\right)} Z_{n} \frac{\sin \left(\frac{z_{n} x}{L}\right) e^{y_{n} T_{1}-a(L-x)}}{y-y_{n}}+\frac{N_{2}-N_{1}}{f(U \beta+y) y} \\
& \cdot \frac{\left[(\beta-a-b) e^{a(L+x)+b(x-L)}-(\beta-a+b) e^{a(L+x)+b(L-x)}-2 e^{a x}(-2 a+\beta) \sinh (b x)\right]}{2 e^{a L}((\beta-a) \sinh (b L)+b \cosh (b L))}
\end{aligned}
$$

\section{A2. Response of the Groundwater Table to $N_{1}$}

[69] Equation (A10), considered as a realistic water table profile, is used here as the initial condition when calculating the response of the water table to a recharge $N_{1}$ with duration $T_{1}$. Again, the extended Boussinesq equation will be solved through Laplace transformation. In the Laplace domain, the equation to be solved is now

$$
\begin{aligned}
K f U \beta & \frac{d^{2} F}{d x^{2}}+f U \beta(U-K \beta) \frac{d F}{d x}-f U \beta(U \beta+y) F \\
= & -U \beta \frac{N_{1}}{y}-N_{0}+N_{0} \frac{2(\beta-a) e^{\beta L}+(2 a-\beta)}{2(\beta-a) e^{\beta L}} e^{(2 a-\beta) x} \\
& +N_{0} \frac{(-2 a+\beta) e^{\beta x}}{2(\beta-a) e^{\beta L}}
\end{aligned}
$$

The expression for $F$ is

$$
\begin{aligned}
& \qquad \begin{aligned}
F= & \frac{N_{1}-N_{0}}{f(U \beta+y) y} \\
& \cdot \frac{(\beta-a-b) e^{a(L+x)+b(x-L)}-(\beta-a+b) e^{a(L+x}}{2 e^{a L}((\beta-a) \sinh (b L)} \\
& +\frac{U \beta N_{1}+N_{0} y}{U \beta f(U \beta+y) y}+\frac{N_{0}}{U \beta f y} \frac{(2 a-\beta)}{2(\beta-a) e^{\beta L}} e^{\beta x} \\
& -\frac{N_{0}}{U \beta f} \frac{2(\beta-a) e^{\beta L}+(2 a-\beta)}{2 y(\beta-a) e^{\beta L}} e^{(2 a-\beta) x}
\end{aligned} \\
& \text { After inverse transformation, the obtained water table height } \\
& \text { is given by } \\
& h_{1}(x, t)=h_{0}\left(x, N_{1}\right)+2 \sum_{n=1}^{\infty} \frac{N_{1}-N_{0}}{f\left(U \beta+y_{n}\right)} Z_{n} \sin \left(\frac{z_{n} x}{L}\right) e^{y_{n} t-a(L-x)}
\end{aligned}
$$$$
\frac{(\beta-a-b) e^{a(L+x)+b(x-L)}-(\beta-a+b) e^{a(L+x)+b(L-x)}-2 e^{a x}(-2 a+\beta) \sinh (b x)}{2 e^{a L}((\beta-a) \sinh (b L)+b \cosh (b L))}
$$

which finally yields the following groundwater profile:

$$
\begin{aligned}
h_{2}(x, t)= & h_{0}\left(x, N_{2}\right)+2 \sum_{n=1}^{\infty} \frac{\left(N_{1}-N_{0}\right) e^{y_{n} T_{1}}+\left(N_{2}-N_{1}\right)}{f\left(U \beta+y_{n}\right)} \\
& \cdot Z_{n} \sin \left(\frac{z_{n} x}{L}\right) e^{y_{n} t-a(L-x)}
\end{aligned}
$$

\section{A4. Response of the Groundwater Table to $\boldsymbol{N}_{\boldsymbol{m}}$}

[71] In general, it can easily be proven that the solution to the extended Boussinesq equation for a temporally varying recharge rate can be written as

$$
\begin{aligned}
h(x, t)= & h_{0}\left(x, N_{m}\right)+2 \sum_{n=1}^{\infty}\left[\sum_{i=1}^{m}\left(N_{i}-N_{i-1}\right) e^{y_{n} \sum_{j=i}^{m-1} T_{j}}\right] \\
& \cdot \frac{Z_{n}}{f\left(U \beta+y_{n}\right)} \sin \left(\frac{z_{n} x}{L}\right) e^{y_{n} t-a(L-x)}
\end{aligned}
$$

This expression calculates the transient profile of the groundwater table, responding to a constant recharge rate $N_{1}$, with initial condition the steady state water table profile obtained with recharge $N_{0}$.

\section{A3. Response of the Groundwater Table to $\boldsymbol{N}_{\mathbf{2}}$}

[70] At the onset of a new recharge rate $N_{2}\left(t=T_{1}\right)$, the groundwater profile is given by equation (A13) evaluated at $t=T_{1}$. Again, the extended Boussinesq equation is solved through Laplace transformation. The resulting ODE is

$$
\begin{aligned}
& K f U \beta \frac{d^{2} F}{d x^{2}}+f U \beta(U-K \beta) \frac{d F}{d x}-f U \beta(U \beta+y) F \\
&=--U \beta \frac{N_{2}}{y}-N_{1}+N_{1} \frac{(-2 a+\beta) e^{\beta x}}{2(\beta-a) e^{\beta L}} \\
&+N_{1} \frac{2(\beta-a) e^{\beta L}-(-2 a+\beta)}{2(\beta-a) e^{\beta L}} e^{(2 a-\beta) x} \\
&-2 f U \beta \sum_{n=1}^{\infty} \frac{N_{1}-N_{0}}{f\left(U \beta+y_{n}\right)} Z_{n} \sin \left(\frac{z_{n} x}{L}\right) e^{y_{n} T_{1}-a(L-x)}
\end{aligned}
$$

where $T_{0}=0$. The first term in this equation represents the steady state condition, while the second term represents the transient condition. We define $\mathcal{T}_{i}$ as follows:

$$
\mathcal{T}_{i}=\sum_{j=i}^{m-1} T_{j}
$$

The summation equals zero when $m-1$ is lower than one.

\section{A5. Calculation of the Groundwater Outflow}

[72] The linearized Darcy equation (equation (7)) represents the relationship between the discharge and the height of the water table. Application of equation (7) yields the groundwater flux per unit width. Multiplication by $-2 W_{0}$ (the minus sign is to render base flow values positive, and $2 W_{0}$ accounts for the length of the drainage network with drainage from both banks) and setting $x$ equal to zero leads to the base flow rate of the catchment:

$$
\begin{aligned}
Q_{b}(t)= & A N_{m}+\left(N_{m}-N_{m-1}\right)\left[\sum_{n=1}^{\infty} \frac{4 W_{0} K Z_{n}}{U \beta+y_{n}} \frac{z_{n}}{L} e^{y_{n} t-a L}\right] \\
& +\sum_{i=1}^{m-1}\left(N_{i}-N_{i-1}\right)\left[\sum_{n=1}^{\infty} \frac{4 W_{0} K Z_{n}}{U \beta+y_{n}} \frac{z_{n}}{L} e^{y_{n}\left(t+\mathcal{T}_{i}\right)-a L}\right]
\end{aligned}
$$


[73] Acknowledgments. The author would like to express his gratitude to Willem Defloor from the Department Water of the Ministry of the Flemish Community for providing the discharge and precipitation data. We would also like to thank three anonymous reviewers for their helpful comments, remarks, and suggestions.

\section{References}

Bear, J. (1972), Dynamics of Fluids in Porous Media, Elsevier, New York. Beven, K. J., and M. J. Kirkby (1979), A physically based, variable contributing area model of basin hydrology, Hydrol. Sci. Bull., 24(1), 43-69.

Boughton, W. C. (1993), A hydrograph-based model for estimating the water yield of ungauged catchments, paper presented at Hydrology and Water Resources Symposium, Inst. of Eng., Newcastle, N. S. W., Australia.

Brutsaert, W. (1994), The unit response of groundwater outflow from a hillslope, Water Resour. Res., 30(10), 2759-2763.

Brutsaert, W., and J. L. Nieber (1977), Regionalized drought flow hydrographs from a mature glaciated plateau, Water Resour. Res., 13(3), 637-643.

Camporese, M., C. Paniconi, M. Putti, and P. Salandin (2009), Ensemble Kalman filter data assimilation for a process-based catchment scale model of surface and subsurface flow, Water Resour. Res., 45, W10421, doi:10.1029/2008WR007031.

Chapman, T. (1995), Comment on "The unit response of groundwater outflow from a hillslope" by Wilfried Brutsaert, Water Resour. Res., $31(9), 2377-2378$

Chapman, T. G. (2005), Recharge-induced groundwater flow over a plane sloping bed: Solutions for steady and transient flow using physical and numerical models, Water Resour. Res., 41, W07027, doi:10.1029/ 2004WR003606.

Chapman, T., and A. Maxwell (1996), Baseflow separation-Comparison of numerical methods with tracer experiments, paper presented at Hydrology and Water Resources Symposium, Inst. of Eng., Hobart, Tasmania, Australia.

Chapman, T. G., and G. Ong (2006), A new equation for shallow groundwater flow over a curved impermeable boundary: Numerical solutions and laboratory tests, Water Resour. Res., 42, W03427, doi:10.1029/ 2005WR004437.

Dai, Y. J., et al. (2003), The Common Land Model, Bull. Am. Meteorol. Soc., 84(8), 1013-1023.

Desilets, S. L. E., T. P. A. Ferré, and B. Ekwurzel (2008), Flash flood dynamics and composition in a semiarid mountain watershed, Water Resour. Res., 44, W12436, doi:10.1029/2007WR006159.

Famiglietti, J. S., and E. F. Wood (1994), Multiscale modeling of spatially variable water and energy balance processes, Water Resour. Res., 30(11), 3061-3078.

Fan, Y., and R. L. Bras (1998), Analytical solutions to hillslope subsurface storm flow and saturation overland flow, Water Resour. Res., 34(4), 921-927.

Furey, P. R., and V. K. Gupta (2001), A physically based filter for separating base flow from streamflow time series, Water Resour. Res., 37(11), $2709-2722$.

Furey, P. R., and V. K. Gupta (2003), Test of two physically based filters for base flow separation, Water Resour. Res., 39(10), 1297, doi:10.1029/ 2002WR001621.

Harman, C., and M. Sivapalan (2009a), Effects of hydraulic conductivity variability on hillslope-scale shallow subsurface flow response and storage-discharge relations, Water Resour. Res., 45, W01421, doi:10.1029/ 2008WR007228.

Harman, C., and M. Sivapalan (2009b), A similarity framework to assess controls on shallow subsurface flow dynamics in hillslopes, Water Resour. Res., 45, W01417, doi:10.1029/2008WR007067.

Harman, C. J., M. Sivapalan, and P. Kumar (2009), Power law catchmentscale recessions arising from heterogeneous linear small-scale dynamics, Water Resour. Res., 45, W09404, doi:10.1029/2008WR007392.

Hasan, S., P. A. Troch, P. W. Bogaart, and C. Kroner (2008), Evaluating catchment-scale hydrological modeling by means of terrestrial gravity observations, Water Resour. Res., 44, W08416, doi:10.1029/ 2007WR006321.

Hilberts, A. G. J., E. E. van Loon, P. A. Troch, and C. Paniconi (2004), The hillslope-storage Boussinesq model for non-constant bedrock slope, J. Hydrol., 291(3-4), 160-173.

Huyck, A. A. O., V. R. N. Pauwels, and N. E. C. Verhoest (2005), A base flow separation algorithm based on the linearized Boussinesq equation for complex hillslopes, Water Resour. Res., 41, W08415, doi:10.1029/ 2004WR003789.
Jakeman, A. J., and G. M. Hornberger (1993), How much complexity is warranted in a rainfall-runoff model?, Water Resour. Res., 29(12), $2637-2649$.

Kirchner, J. W. (2009), Catchments as simple dynamical systems: Catchment characterization, rainfall-runoff modeling, and doing hydrology backward, Water Resour. Res., 45, W02429, doi:10.1029/2008WR006912.

Koussis, A. (1992), A linear conceptual subsurface storm flow model, Water Resour. Res., 28(4), 1047-1052.

Liang, X., D. P. Lettenmaier, E. F. Wood, and S. J. Burges (1994), A simple hydrologically based model of land surface water and energy fluxes for general circulation models, J. Geophys. Res., 99(D7), 14,415-14,428.

Liu, Y. H., N. J. Fan, S. Q. An, X. H. Bai, F. D. Liu, Z. Xu, Z. S. Wang, and S. R. Liu (2008), Characteristics of water isotopes and hydrograph separation during the wet season in the Heishu River, China, J. Hydrol., $353(3-4), 314-321$.

Lockington, D. A. (1997), Response of unconfined aquifer to sudden change in boundary head, J. Irrig. Drain. Eng., 123(1), 24-27.

Lyne, V. D., and M. Hollick (1979), Stochastic time-variable rainfall-runoff modelling, paper presented at Hydrology and Water Resources Symposium, Inst. of Eng., Canberra.

Lyon, S. W., S. L. E. Desilets, and P. A. Troch (2008), Characterizing the response of a catchment to an extreme rainfall event using hydrometric and isotopic data, Water Resour. Res., 44, W06413, doi:10.1029/ 2007WR006259.

Nakaya, S., K. Uesugi, Y. Motodate, I. Ohmiya, H. Komiya, H. Masuda, and M. Kusakabe (2007), Spatial separation of groundwater flow paths from a multi-flow system by a simple mixing model using stable isotopes of oxygen and hydrogen as natural tracers, Water Resour. Res., 43, W09404, doi:10.1029/2006WR005059.

Paniconi, C., and E. F. Wood (1993), A detailed model for simulation of catchment scale subsurface hydrologic processes, Water Resour. Res., 29(6), 1601-1620.

Paniconi, C., P. A. Troch, E. E. van Loon, and A. Hilberts (2003), Hillslopestorage Boussinesq model for subsurface flow and variable source areas along complex hillslopes: 2. Intercomparison with a three-dimensional Richards equation model, Water Resour. Res., 39(11), 1317, doi:10.1029/2002WR001730.

Pauwels, V. R. N., N. E. C. Verhoest, and F. P. De Troch (2002), A metahillslope model based on an analytical solution to a linearized Boussinesqequation for temporally variable recharge rates, Water Resour. Res., 38(12), 1297, doi:10.1029/2001WR000714.

Polubarinova-Kochina, P. Y.-A. (1962), Theory of Groundwater Movement, translated from Russian by R. J. M. De Wiest, 613 pp., Princeton Univ. Press, Princeton, N. J.

Richards, L. (1931), Capillary conduction of liquids through porous media, Physics, 1, 318-333.

Rupp, D. E., and J. S. Selker (2005), Drainage of a horizontal Boussinesq aquifer with a power law hydraulic conductivity profile, Water Resour. Res., 41, W11422, doi:10.1029/2005WR004241.

Rupp, D. E., and J. S. Selker (2006a), On the use of the Boussinesq equation for interpreting recession hydrographs from sloping aquifers, Water Resour. Res., 42, W12421, doi:10.1029/2006WR005080.

Rupp, D. E., and J. S. Selker (2006b), Information, artifacts, and noise in $d Q / d t-Q$ recession analysis, Adv. Water Resour., 29(2), 154-160.

Swenson, S., J. Famiglietti, J. Basara, and J. Wahr (2008), Estimating profile soil moisture and groundwater variations using GRACE and Oklahoma Mesonet soil moisture data, Water Resour. Res., 44, W01413, doi:10.1029/2007WR006057.

Szilagyi, J., M. B. Parlange, and J. Albertson (1998), Recession flow analysis for aquifer parameter determination, Water Resour. Res., 34(7), $1851-1857$.

Tetzlaff, D., and C. Soulsby (2008), Sources of baseflow in larger catchments-Using tracers to develop a holistic understanding of runoff generation, J. Hydrol., 359(3-4), 287-302.

Tetzlaff, D., C. Soulsby, S. Waldron, I. A. Malcolm, P. J. Bacon, S. M. Dunn, A. Lilly, and A. F. Youngson (2007), Conceptualization of runoff processes using a geographical information system and traces in a nested mesoscale catchment, Hydrol. Processes, 21(10), 1289-1307.

Troch, P. A., F. P. De Troch, and W. Brutsaert (1993), Effective water table depth to describe initial conditions prior to storm rainfall in humid regions, Water Resour. Res., 29(2), 427-434.

Troch, P. A., J. A. Smith, E. F. Wood, and F. P. De Troch (1994), Hydrologic controls of large floods in a small basin: Central Appalachian case study, J. Hydrol., 156(1-4), 285-309.

Troch, P., E. van Loon, and A. Hilberts (2002), Analytical solutions to a hillslope-storage kinematic wave equation for subsurface flow, $A d v$. Water Resour., 25(6), 637-649. 
Troch, P. A., C. Paniconi, and E. E. van Loon (2003), Hillslope-storage Boussinesq model for subsurface flow and variable source areas along complex hillslopes: 1. Formulation and characteristic response, Water Resour. Res., 39(11), 1316, doi:10.1029/2002WR001728.

Troch, P. A., A. H. van Loon, and A. G. J. Hilberts (2004), Analytical solution of the linearized hillslope-storage Boussinesq equation for exponential hillslope width functions, Water Resour. Res., 40, W08601, doi:10.1029/2003WR002850.

Verhoest, N. E. C., and P. A. Troch (2000), Some analytical solutions of the linearized Boussinesq equation with recharge for a sloping aquifer, Water Resour. Res., 36(3), 793-800.

Westhoff, M. C., H. H. G. Savenije, W. M. J. Luxemburg, G. S. Stelling, N. C. van de Giesen, J. S. Selker, and S. Uhlenbrook (2007), A distrib- uted stream temperature model using high resolution temperature observations, Hydrol. Earth Syst. Sci., 11(4), 1469-1480.

Wood, E. F. (1991), Global scale hydrology: Advances in land surface modeling, Rev. Geophys., 29, 193-201.

V. R. N. Pauwels, Laboratory of Hydrology and Water Management, Ghent University, Coupure links 653, B-9000 Ghent, Belgium. (valentijn. pauwels@ugent.be)

P. A. Troch, Department of Hydrology and Water Resources, University of Arizona, Tucson, AZ 85721, USA. 